\title{
Haciendo encajar las piezas en las causativas analíticas con hacer ${ }^{1}$
}

\section{Assembling the pieces of the puzzle of analytic causatives with hacer}

\author{
Margot Vivanco \\ Universidad de Castilla-La Mancha
}

Resumen. Dado que tanto los predicados complejos como los verbos de reestructuración conforman clases heterogéneas — dentro de una sola lengua y también a nivel tipológico—-, resulta necesario estudiar cada caso en detalle. Así, este trabajo se centra en las causativas analíticas con hacer del español, ofreciendo un pormenorizado panorama general de sus propiedades que permitirá esclarecer algunos de los puntos más controvertidos para su análisis, como el tamaño del infinitivo y la posición del causee. Concretamente, se argumentará que el causee se encuentra en un SVoz y que, por lo tanto, el infinitivo no proyecta un SV escueto, sino que cuenta con una estructura funcional que incluye SVoz, SModalidad deóntica y SAsp.

Palabras clave: causativas analíticas, predicados complejos, asignación de caso, orden de palabras.

Abstract. Since both complex predicates and restructuring verbs constitute heterogeneous classes -within one single language and also cross-linguistically - , a detailed study of each case is in order. This paper focuses on Spanish analytic causatives with hacer ('make'), providing a detailed

Data de recepción: 30-07-2017 - Data de aceptación: 17-11-2017.

Este estudio ha sido realizado en el marco del grupo de investigación Gramática Teórica del Español (930590) de la Universidad Complutense de Madrid. Quiero expresar mi gratitud a Cristina Sánchez López, Antonio Fábregas, Ignacio Bosque, M. Jesús Fernández Leborans, Víctor Acedo Matellán y a los revisores anónimos de la revista, pues sus comentarios han contribuido significativamente a mejorar este trabajo. 
overview of their properties that will shed light on some of their most controversial syntactic puzzles, i.e. the size of the infinitive projection and the position of the causee. More specifically, it will be argued that the causee is placed on a VoiceP and, consequently, that the infinitive does not project a bare vP, but a higher functional structure, including VoiceP, deontic MoodP and AspP. Keywords: analytic causatives, complex predicates, case, word order.

\section{INTRODUCCIÓN}

En las Causativas Analíticas (CCAA) del español (1) y otras lenguas, hacer y el infinitivo conforman un Predicado Complejo (PC), pues exhiben un fenómeno de reducción tal que dos eventos quedan semifusionados sintácticamente en una oración simple, conservándose, no obstante, ambos núcleos.

(1) Nerea hizo llorar a sus enemigos.

Como señala Svenonius (2008), prácticamente cualquier predicado con dos partes puede clasificarse como «complejo»: las construcciones resultativas, los verbos en serie de las lenguas africanas y austronesias, los verbos compuestos de las lenguas sudasiáticas, las construcciones con verbo ligero, los verbos de reestructuración, etc. Todos ellos se caracterizan por exhibir la estructura funcional de un predicado simple, con un único sujeto, pese a encerrar una estructura argumental compleja, con dos núcleos que aportan argumentos (Butt 1995). Es, de hecho, una condición sine qua non que la construcción sea monoclausal ${ }^{2}$, lo cual solo es posible si el verbo principal toma como complemento una estructura menor que ST ( $c f r$. Baker 1988; Wurmbrand 2001; Tubino Blanco et alii 2014). De los dos elementos que conforman el PC, uno ha de ser un verbo y combinarse con T; normalmente este es el que denota el evento ${ }^{3}$, lleva la carga funcional ${ }^{4} \mathrm{y}$ se encuentra en la posición estructural más elevada. En cambio, el otro elemento puede ser un verbo en forma no personal, un sustantivo, un

2 En lenguas como el japonés, las dos unidades que integran el PC quedan amalgamadas morfológicamente mediante un proceso de incorporación sintáctica (cfr. Baker 1988). Se considera que estas causativas sintéticas tienen la misma estructura que las analíticas, siendo la materialización —independiente o «amalgamada»— de los núcleos la única diferencia entre ellas (cfr. Harley 1995).

3 Si el segundo elemento no es un verbo, el primer elemento es el que aporta eventividad al predicado complejo - Cristina se puso nerviosa -, mientras que si el segundo elemento es también un verbo, ambos aportan contenido eventivo.

4 Si este elemento aporta exclusivamente contenido funcional, es un auxiliar. 
adjetivo, etc., tiene mayor carga semántica y se encuentra en la posición estructural inferior (cfr. Svenonius 2008).

Los verbos causativos y los de control se consideran verbos de reestructuración (cfr. Wurmbrand 2001; Cardinaletti 2004; Campanini \& Pitteroff 2013), si bien esta operación no parece funcionar igual en todos los casos, ni en todas las lenguas.

Estos verbos portan la información funcional aun conservando significado léxico $\mathrm{y}$, a diferencia de los ligeros ${ }^{5}$, se caracterizan por no imponer restricciones a sus complementos. Para muchos autores (Rizzi 1978, 1982; Haegeman \& van Riemdijk 1986; von Stechow 1990), la reestructuración es un proceso mediante el que dos oraciones quedan reducidas a una, lo cual presupone la existencia de dos límites oracionales en un primer estadio de la derivación, y la posterior supresión del inferior. Otros autores (Rizzi 1982; Baker 1988; Burzio 1986) proponen que el límite inferior — SC o similar - es transparente o defectivo, lo que le permite formar un PC con el superior. No obstante, este estudio parte de la hipótesis de que la construcción causativa comienza directamente como monoclausal en la derivación, es decir, el verbo inferior no proyecta en ningún momento ST o SC y esto es lo que permite que forme un PC con el verbo superior, sin necesidad de ninguna operación específica de reestructuración o reducción ( $c f r$. Wurmbrand 2001, 2004, 2007) ${ }^{6}$. Respecto al estatus de hacer en español como verbo léxico pleno o elemento funcional, se argumentará, siguiendo a autores como Cardinaletti (2004), que se trata de un elemento «cuasifuncional», por sus propiedades sintáctico-semánticas mixtas.

Este trabajo aborda dos de los principales problemas del análisis de las CCAA como PPCC de reestructuración. El primero de ellos (apartado 2) atañe a la posición del causee, para el cual se defenderá un análisis en SVoz frente a aquellas propuestas que lo ubican bien en una aplicativa (Campanini \& Pitteroff 2013), o bien en un especificador a la derecha (Folli \& Harley 2007). Así mismo, se discutirán otras cuestiones relacionadas con su interpretación temática. El segundo problema (apartado 3) concierne al tamaño de la proyección del infinitivo, pues se ha defendido que se trata de un SV escueto (Folli \& Harley 2007), que incluye Voz (Campanini \& Pitteroff 2013) o incluso que contiene cierta estructura funcional — ST o SC - (Baker 1988). Los datos presentados en los siguientes apartados demostrarán que la estructura del

$5 \quad$ Por ejemplo, verbos ligeros como ponerse, volverse o hacerse en español imponen serias restricciones a sus complementos, basadas en la dicotomía entre predicados de individuo y de estadio —*Ponerse fumador, *Hacerse nervioso - ; en cambio, el verbo hacer puede subordinar cualquier tipo de infinitivo.

$6 \quad$ Sobre la reestructuración véase Rizzi (1978, 1982), Aissen \& Perlmutter (1983), Roberts (1997), Wurmbrand (2001) y Cinque (2006), entre otros. 
infinitivo llega hasta SAsp, de manera que abarca SVoz y SM-deóntico, pero excluye cualquier proyección superior — como SM-epistémico o ST- El apartado 4 presenta una propuesta de análisis de reestructuración basada en una versión del tradicional predicate raising $\mathrm{y}$, finalmente, el apartado 5 resume las conclusiones fundamentales de este estudio.

\section{EL CAUSEE}

Se empleará el término causee $e^{7}$ para referir al argumento que funcionaría como sujeto del infinitivo si este estuviera flexionado, es decir, a su Argumento Externo (AE) si es un verbo transitivo o inergativo, o a su Argumento Interno (AI) si es inacusativo.

Se comenzará por abordar su interpretación temática en el apartado 2.1. A este respecto, se estudiará cómo repercute en las distintas interpretaciones semánticas de las CCAA — causación indirecta y obligación — y si estas son o no el reflejo de posibles diferencias sintácticas. El apartado 2.1.2. trata de la relación entre el orden de palabras - anteposición del causee — y la semántica de las CCAA, adelantando la discusión del apartado 2.2. sobre la posición sintáctica del causee pues, dado que en el orden no marcado este sigue no solo al verbo sino a todo el SV, resulta imprescindible dilucidar su posición de base. Tras descartar otros análisis previos, se establecerá que el causee se encuentra en SVoz, lo cual supone un primer paso para resolver la cuestión del tamaño de la proyección del infinitivo en el apartado 3 así como para proponer una versión del predicate raising en el apartado 4, ya que el SV ha de desplazarse por encima de su AE.

\subsection{La interpretación temática del causee}

\subsubsection{Las lecturas de las CCAA}

Considérese la ambigüedad de la siguiente oración:

(2) David Bowie hizo cantar a Víctor.

i. Causó indirectamente que él cantara, por ejemplo inspirándole (LCM).

ii. Le obligó a cantar (LOBL).

El anglicismo se emplea para evitar el término causado, que se reserva para aludir al (sub-)evento provocado por el (sub-)evento causante. 
En la lectura de causa mediata (LCM), X provoca que $Y$ haga algo o que le suceda algo. Esta es la única interpretación posible cuando el sujeto de hacer es inanimado (3a). En la lectura de obligación (LOBL), X obliga a Y a hacer algo. Las condiciones para obtener esta lectura son más restrictivas, pues ambos participantes han de ser, además de animados, agentivos (3b):

(3) a. La necesidad económica hizo a Víctor cantar en el metro (LCM, \#LOBL).

b. Andrea hizo caer a Diana (LCM, \#LOBL).

Folli y Harley (F\&H) (2007) defienden que a estas diferentes interpretaciones subyacen diferentes configuraciones sintácticas. Las autoras señalan que ninguna de las dos lecturas puede obtenerse si el AE del infinitivo es una causa y extraen de (4a) una generalización sintáctica: hacer puede tomar como complemento un $\mathrm{v}_{\mathrm{DO}}$-con lo que se obtiene la LOBL si el sujeto de hacer también es agentivo- o un $\mathrm{v}_{\mathrm{BECOME}}$, pero nunca un $\mathrm{v}_{\text {CAUSE }}$. Recordemos que, de acuerdo con la teoría de los «sabores (flavours) de $v$ pequeña» (Harley 1995; Folli \& Harley 2005), los rasgos en $\mathrm{v}$ determinan la presencia o la ausencia de $\mathrm{AE}-\operatorname{los} \mathrm{v}_{\mathrm{DO}} \mathrm{y}_{\mathrm{CAUSE}}$ de los verbos transitivos e inergativos frente al $\mathrm{v}_{\mathrm{BECOME}}$ de los inacusativos - $\mathrm{y}$ también el papel temático de este $-\mathrm{v}_{\mathrm{DO}}$, agentivo, frente $\mathrm{a}_{\mathrm{CAUSE}}$, causante-.

(4) a. Gianni ha fatto rompere la finestra *al ramo / a María. Gianni ha hecho romper la ventana a.la rama a María.

(F\&H 2007: 201)

b. *Pepe / La tormenta hizo a la rama romper la ventana.

Nótese, sin embargo, que esta hipótesis no es coherente con la estructura de las causativas léxicas, pues aceptarla obligaría a establecer una diferencia sintáctica entre las oraciones de (5a-5b), fundamentada únicamente en la animacidad del AE, a fin de que la oración de (6b) sea gramatical, frente a (6a):

(5) a. El viento rompió la ventana. $\rightarrow\left[\mathbf{v}_{\mathrm{CAUSE}}\left[\mathrm{v}_{\mathrm{BE}}\right]\right]$

b. Diana rompió la ventana. $\rightarrow\left[\mathbf{v}_{\mathrm{DO}}\left[\mathrm{v}_{\mathrm{BE}}\right]\right]$

(6) a. *Diana / la tormenta hizo al viento romper la ventana.

$\rightarrow\left[\right.$ hacer $\left.\left[\mathbf{v}_{\mathrm{CAUSE}}\left[\mathrm{v}_{\mathrm{BE}}\right]\right]\right]$

b. Andrea / el calor hizo a Diana romper la ventana.

$\rightarrow\left[\right.$ hacer $\left.\left[\mathbf{v}_{\mathbf{D O}}\left[\mathrm{v}_{\mathrm{BE}}\right]\right]\right]$ 
De acuerdo con Hale \& Keyser (1993), Higginbotham (2000), Cuervo (2003), Alexiadou et alii (2006, 2015), Nash (2006), Ramchand (2008) o Schäfer (2008) los AAEE de los verbos causativos del tipo romper obtienen configuracionalmente el papel temático de causante por encontrarse en una estructura causativa, es decir, una estructura con dos subeventos (7a) tal que uno (dinámico) causa el otro (estativo). $\mathrm{Si}$ el SN es humano, es posible además atribuirle propiedades agentivas, pero no por ello deja de ser un causante (véase también Cano Aguilar 1977: 333-335). Del mismo modo, el AE de hacer se encuentra también en una estructura con dos eventos, tal que uno causa el otro, siendo la única diferencia el hecho de que, en este caso, el evento subordinado no es necesariamente un estado. Más concretamente, el AE de hacer se interpreta como «causa indirecta» porque el evento causante y el causado no están amalgamados (Nedjalkov \& Silnitsky 1973; Levin \& Rappaport Hovav 1999), es decir, están materializados por unidades léxicas independientes. Así, la LCM es la lectura por defecto, mientras que la LOBL no es más que un efecto semántico que se produce si tanto el causante como el causee son animados y, por tanto, capaces de tener volición y control ${ }^{8}$.
(7) a. [causante [ev. dinámico [estado]]]
b. [causante [ev. dinámico hacer [causante [ev. dinámico romper [estado]]]]]

En consecuencia, si hacer puede subordinar verbos causativos como romper ( $7 b)$, no puede haber una restricción que impida la presencia de un causante en la oración de infinitivo. Así pues, la inaceptabilidad de (4) no se debe en realidad a agramaticalidad sintáctica, sino a una anomalía semántica relacionada con la interpretación de las cadenas causales y con cómo nuestro conocimiento del mundo nos dice que ciertas entidades no pueden formar parte de ellas, pues no pueden estar sujetas a condicionamiento alguno ( $c f r$. Neeleman \& van de Koot 2012).

\subsubsection{El orden de palabras y sus posibles repercusiones interpretativas}

En español, a diferencia de lo que sucede en otras lenguas, el causee puede preceder o seguir al infinitivo - Nerea hizo a sus enemigos llorar frente a Nerea hizo llorar a sus enemigos - En este apartado descartaremos la idea de que el orden de palabras determine la elección entre las lecturas estudiadas en el anterior (LOBL y LCM) y plantearemos, en cambio, que está relacionado con cuestiones de procesamiento sintáctico.

Ello no es óbice para que el AE de hacer siga desempeñando el papel de causante, pues la obligación es una forma de causar un evento. De ahí procede precisamente la ambigüedad de (2). 
Torrego (2010) relaciona la anteposición del causee con la LOBL. Argumenta que la interpretación semántica del $\mathrm{AE}$ de hacer como causa o agente está determinada por la posición pre- o posverbal del causee: si este es preverbal, el AE de hacer solo puede interpretarse como agente, por lo que no puede ser inanimado (8) y solo la lectura de obligación está disponible — siempre y cuando el causee también sea agentivo- - En cambio, si el causee es posverbal, el AE de hacer puede interpretarse indistintamente como agente o como causa, de modo que tanto la LOBL como la LCM estarían disponibles. En otras palabras, la LOBL permite, según Torrego, dos posiciones para el causee, mientras que la LCM permite exclusivamente la posverbal":

(8) a. ? La recesión ha hecho a la atleta perder el trabajo.

(Torrego 2010: 449) ${ }^{10}$

b. La recesión le ha hecho perder su trabajo a la atleta.

En (9) se recogen más ejemplos en los que un causee preverbal parece bloquear la interpretación del sujeto principal como causa. (9b) es anómala sea cual fuere el AE de hacer, pues la LOBL es imposible con un causee inanimado, y la LCM parece empeorar notablemente con un causee preverbal. En cambio, en (10) se observa que estos ejemplos mejoran significativamente con la mera posposición del causee.

(9) a. David Bowie / ?la necesidad económica hizo a Víctor cantar.

b. ?Fernando / ?el calor hizo el rosal florecer.

(10) a. David Bowie / la necesidad económica hizo trabajar a Víctor.

b. Fernando / el calor hizo florecer el rosal.

No obstante, nótese que nuestros ejemplos de (9) contienen, como los de Torrego en (8), interrogaciones en lugar de asteriscos, lo cual indica que son dudosamente aceptables en un contexto neutro; sin embargo, con la entonación adecuada, la anteposición puede relacionarse con la estructura informativa, de manera que el causee funcione bien como tópico o bien como foco contrastivo. El ejemplo de (11a) muestra, no obstante, que la LCM sí admite causees preverbales, lo que contradice la afirmación de Torrego (2010). Un factor que contribuye a la aceptabilidad de este orden es la animacidad, razón por la que esta autora ve un vínculo entre esta posición y la LOBL. Nótese además que la posposición del causee en este ejemplo resulta anómala (11b):

\footnotetext{
9 Un revisor anónimo observa que perder no es un verbo agentivo, lo que descarta la LOBL en (8) independientemente del orden de palabras.

$10 \quad$ La interrogación es de Torrego.
} 
(11) a. El hambre hizo a Victor plantearse robar comida del mercado.

b. ?El hambre hizo plantearse robar comida del mercado a Víctor.

Parece, pues, que la anteposición se ve favorecida por factores relacionados con el procesamiento, volviéndose casi obligatoria en contextos en los que el peso sintáctico del SV (11), el encadenamiento de varios infinitivos (12), o el de varios SSDD (13) hacen necesario reordenar las palabras para facilitar la comprensión:

(12) a. Yuridia hizo a Maca hacer a Luis hacer a Javi hacer a Alex traer vodka.

b. *Yuridia hizo hacer hacer hacer traer vodka a Maca a Luis a Javi a Alex.

c. *Yuridia hizo hacer a Maca hacer traer vodka a Luis a Javi.

(13) a. Yuridia hizo a Alex cortarle el flequillo a Macarena.

b. *Yuridia hizo cortarle el flequillo a Macarena a Alex.

La anteposición del causee, además de contribuir al procesamiento de la secuencia, es una posición marcada en la que bloquea sintácticamente el ascenso del clítico de objeto:

(14) *La hizo a Leonardo construir.

(Treviño 1992: 316)

Del mismo modo, los adverbios orientados al agente pueden referirse tanto al sujeto de hacer como al causee si este último es posverbal (15a), mientras que, si es preverbal, han de orientarse a él exclusivamente (15b):

(15) a. El director ${ }_{i}$ hizo saludar a los niños amablemente $_{\mathrm{ij}}$.

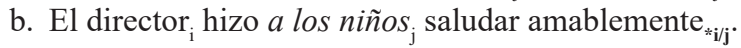

En definitiva, los datos presentados indican que la posición no marcada del causee es la posverbal ${ }^{12}$, pero no que la preverbal tenga incidencia alguna sobre la interpretación temática del causante (contra Torrego 2010). En cambio, la anteposición

11 Evitando así problemas de incrustación en el centro (center-embedding). Se ha oberservado en la bibliografía que la posición preverbal está reservada a los causees, pues no puede haber anteposición del objeto ( $c f r$. Ordóñez \& Saab 2017); en relación con ejemplos como el de (13), resulta evidente que esto contribuye a evitar ambigüedades respecto a quién le corta el flequillo a quién gracias a respetar un orden canónico S-V-O: Hizo al abuelo recoger a los niños del colegio frente a *Hizo a los niños recoger al abuelo del colegio.

12 Véase Ordóñez \& Saab (2017) para más argumentos a favor de considerar los causees preverbales como una posición derivada frente al orden posverbal básico. 
responde a factores relacionados con el procesamiento sintáctico y, posiblemente, con la estructura informativa.

En el siguiente apartado se planteará una hipótesis sobre la posición de base del causee; sin embargo, dilucidar cuál es exactamente la posición a la que se desplaza cuando aparece antepuesto queda fuera de los propósitos de este trabajo. A este respecto cabe conjeturar, siguiendo a Ordóñez y Saab (2017), que se trata de un movimiento opcional por revoltijo (scrambling) a una posición superior. Ordóñez y Saab (2017) proponen que tal posición se encontraría en la proyección de hacer y que sería hacer quien atrae opcionalmente al causee por cuestiones relacionadas con el Marcado Diferencial de Objeto (MDO) (veáse también Ciutescu 2013, 2015), de manera similar a lo que ocurre con el movimiento de objeto (object shift; cfr. Ordóñez 1998; Gallego 2013) ${ }^{13}$.

Para terminar este apartado, cabe destacar que la anteposición del causee es obligatoria en rumano — no se admite la posposición—, y es en portugués más frecuente que la posposición ${ }^{14}$ — no se trata del orden marcado- - Por otra parte, este orden de palabras resulta agramatical en italiano, francés y catalán:

(16) a. L-am fãcut pe Ion să cumpere o maşină scumpă

(Ciutescu 2013: 46) Yo hice a Ion comprar un coche caro.

b. A María fez os miúdos ler esse livro.

(Soares da Silva 2012: 526) la María hizo los niños leer ese libro.

c. *Faccio Carlo baciare le ragazze.

(Iglesias Bango 1992: 95) hago Carlo besar las chicas.

d. *Je ferai Jean partir.

(Kayne 1975: 89) yo haré Jean marcharse.

e. El Joan va fer la Maria comprar un llibre. Joan hizo María comprar un libro.

(Ciutescu 2013: 47)

De acuerdo con Soares da Silva (2012), este contraste, junto con otros similares, apunta a que la construcción causativa ha alcanzado distintos grados de gramaticalización en las lenguas romances. El grado máximo es el de la construcción italiana, seguida por la francesa, la española y, finalmente, la portuguesa (siendo estas cuatro

13 De acuerdo con estos autores, la anteposición está restringida al MDO. Nótese a este respecto que, cuando el verbo subordinado es un inacusativo con sujeto inanimado, la posición preverbal del causee resulta muy marcada: La huelga hizo el tren llegar tarde, no el avión; La noticia hizo su rostro palidecer repentinamente.

14 Sobre todo si el infinitivo es transitivo y el causee es animado ( $c f r$. Soares da Silva 2012). Véase nota anterior. 
las lenguas abordadas por el autor). Esto coincide con la idea ya defendida por Comrie (1976) de que el grado de unión entre el verbo causativo y el infinitivo varía entre las distintas lenguas romances, siendo mayor en italiano que en francés y siendo así mismo mayor en francés que en español (véase también Zubizarreta 1985; Moreno Cabrera 1987; Iglesias Bango 1992), lo cual sugiere que el estatus de fare / faire / hacer / fazer puede no ser el mismo y, en definitiva, que el análisis del PC puede sufrir variaciones de una lengua a otra.

Además, solo el portugués y el español cuentan con una variante en la que hacer como verbo pleno subordina un SC (17), opción de la que carecen el italiano y el francés (18). Por su parte, fazer puede en portugués subordinar también un infinitivo flexionado, con un sujeto antepuesto en nominativo (19):

(17) a. David Bowie hizo que Víctor cantara Space Oddity.

b. A Maria faz com que as crianças saiam.

(Soares da Silva 2012: 526) la María hace que los niños salgan.

(18) a. *Je ferais qu'il vienne en train. yo haré que él venga en tren.

b. *Ho fatto che Giovanni usciva. he hecho que Gianni salga.

(C\&P 2013: 47)

(19) A Maria fez els lerem esse livro.

(Soares da Silva 2012: 526) la María hizo ellos.nom. leer ese libro.

Estos y otros contrastes que serán presentados más adelante apuntan a que fare / faire están más gramaticalizados que hacer / fazer y guardan una relación más estrecha con el infinitivo o, en otras palabras, a que fare / faire se han convertido en elementos verdaderamente funcionales a diferencia de hacer, cuyo estatus no pasa de ser «cuasifuncional», como se defenderá en este trabajo.

Los estudios comparativos (cfr. Comrie 1976; Zubizarreta 1985; Haegeman \& van Riemsdijk 1986; Guasti 1996; Ippolito 2000; Soares da Silva 2012; Capanini \& Pitteroff 2013; Ciutescu 2013; Wurmbrand 2015a, entre otros muchos) demuestran, en efecto, que el fenómeno de la reestructuración se produce en distintos grados comparando no solo las distintas lenguas del mundo, sino los distintos verbos de reestructuración de una misma lengua, pues encontramos una gran variación respecto a las tres propiedades típicas de estas construcciones: la pasiva a larga distancia, el ascenso de clíticos y el scrambling. 


\subsection{La posición sintáctica del causee}

A fin de explicar el orden posverbal no marcado, Folli y Harley (2007), siguiendo a Guasti (1996), sitúan a la derecha el especificador que introduce el AE del infinitivo para las CCAA del italiano. No obstante, Campanini y Pitteroff (C\&P) (2013) aducen dos argumentos empíricos en contra de este análisis. En primer lugar, utilizan los cuantificadores flotantes como diagnóstico para identificar las posiciones, incluida la de base, por las que se ha movido un argumento ( $c f r$. Sportiche 1988). Un análisis con especificadores a la derecha predice erróneamente que un cuantificador asociado al sujeto de hacer debería poder aparecer en posición final:

(20) I professori facevano commentare il libro (*tutti) a Maria (*tutti). los profresores hicieron comentar el libro (todos) a María (todos).

(Guasti 1993: 163, citado en C\&P 2013: 58)

En segundo lugar, Campanini y Pitteroff recuerdan que la motivación original para los especificadores a la derecha fue el orden de palabras de las cláusulas reducidas (21) y señalan que la posposición del sujeto en estos casos no es rígida, pues la inserción de más material sintáctico obliga a su anteposición (22), como es el caso también en nuestros ejemplos de (11-12):

(21) Gianni ha fatto felice Maria.

(C\&P 2013: 58)

Gianni ha hecho feliz María.

(22) ?La sua espressione fa sembrare Giovanni ammalato.

la suya expresión hace parecer Giovanni enfermo.

(Burzio 1986, citado en C\&P 2013: 58)

El español tampoco dispone de argumentos sólidos a favor de un análisis con especificadores a la derecha, especialmente si se tiene en cuenta que la posición posverbal del causee no es rígida, al contrario de lo que sucede en italiano (11a $-E l$ hambre hizo a Victor plantearse robar comida del mercado - frente a 16b — *Faccio Carlo baciare le ragazze-). Además, como veremos más adelante, Folli y Harley proponen que el infinitivo se encuentra en un SV escueto, donde no hay lugar para un SVoz; sin embargo, los datos presentados en el apartado 3 demostrarán que la estructura funcional del infinitivo llega en español hasta SAsp, incluyendo por debajo SM-deóntico y SVoz.

Campanini y Pitteroff (2013) resuelven el problema del orden de palabras en italiano defendiendo que el causee se encuentra en una aplicativa alta (siguiendo a 
Ippolito 2000; Torrego 2010 y Kim 2012). Su principal argumento es que las CCAA rechazan en esta lengua la presencia de otras aplicativas altas (23). El ejemplo de (23b) muestra que esta incompatibilidad no se debe a una violación del principio de distintividad de Richards (2010), según el cual una derivación con dos constituyentes idénticos contiguos colapsa.

(23) a. *Marco ha fatto tagliare l'erba a Martin al suo vicino. (C\&P 2013: 59-60) Marco ha hecho cortar la.hierba a Martin al suyo vecino. 'Marco ha hecho cortar la hierba a Martin a su vecino'.

b. *Marco gli ha fatto tagliare l'erba al suo vicino. Marco le ha hecho cortar la.hierba al suyo vecino. 'Marco le ha hecho cortar(le) la hierba a su vecino'.

En su análisis, el SV subordinado se mueve al especificador del Sv principal, dejado su sujeto atrás, en la línea de Kayne $(1975,2005)$ y otras implementaciones del predicate raising. Posteriormente, fare se desplaza a T, quedando separado del infinitivo.

(24) [SVoice DPCAUSER [vP [vP v- $\sqrt{ } D P]_{i} v-\sqrt{ }$ fare [ApplP DPCAUSEE $\left.\left.\left.\mathrm{t}_{\mathrm{i}}\right]\right]\right]$

(C\&P 2013: 62)

Por otra parte, estos autores proponen que, en alemán, el AE del infinitivo es introducido por Voz, ya que en esta lengua sí es posible insertar aplicativas en las CCAA. Además, el orden de palabras no es ya un problema, pues el verbo aparece en posición final:

(25) Mark Ließ Martin seinem Nachbarn den Rasen mähen.

(C\&P 2013: 59)

Mark dejó Martin su.DAT. vecino la.ACU. hierba cortar.

'Mark dejó a Martin cortar la hierba a su vecino'.

El español admite la inserción de este tipo de aplicativas como el alemán, y a diferencia del italiano, pero comparte con esta última lengua el orden (no marcado) de palabras. La anomalía de (26a) se debe a la violación del principio de distintividad, que se resuelve en (26b-c) mediante la pronominalización del causee o su anteposición, respectivamente:

(26) a. ?Luisa hizo cortarle la hierba a su vecino a Víctor.

b. Luisa le hizo cortarle la hierba a su vecino.

c. Luisa hizo a Víctor cortarle la hierba a su vecino. 
Ahora bien, el problema de esta argumentación radica en que las aplicativas en cuestión no son altas, sino bajas, siguiendo a autoras como Cuervo (2003) o Pylkkänen (2008). Las aplicativas bajas se encuentran en una posición estructural inferior e introducen argumentos no incluidos en la valencia del verbo, los cuales establecen una relación de transferencia dinámica o de posesión estática con el objeto, al que mandan-c: Ramón le cambió una rueda a la bici. Es decir, estos argumentos se relacionan exclusivamente con el objeto, no con el evento completo, y no pueden competir estructuralmente con un AE. Las razones por las que las CCAA del italiano rechazan las aplicativas bajas exceden los límites de esta investigación, si bien se trata de una evidencia más de que estas construcciones cuentan con más restricciones en italiano que en español.

Una verdadera aplicativa alta, en cambio, es aquella que introduce un argumento en la posición estructural más elevada — donde sí puede plantear, a priori, un problema estructural para las CCAA y sus causees -, de manera que este entra en una relación de transferencia o posesión con el evento completo: a Sara le gustan los donuts, a Sol le sobran libros, a Antonio le ocurrieron varias desgracias. Sucede que al observar el comportamiento de estas aplicativas en las CCAA del español se aprecian ciertas anomalías que podrían dar pie a pensar que la hipótesis inicial de Campanini y Pitteroff (2013) es, después de todo, correcta:

(27) a. \#Víctor hizo (a Sara) gustarle las Spice Girls (a Sara).

b. *Cristina hizo (a Antonio) ocurrir(le) varias desgracias (a Antonio).

No obstante, la anomalía de (27a) se debe, una vez más, a la interpretación de las cadenas causales, mientras que la agramaticalidad de (27b) se debe a un problema estructural no relacionado con la posición del causee.

Los verbos psicológicos se consideran estativos y no agentivos, aunque algunos pueden ser eventivos y agentivos ( $c f r$. Marín 2011), y se dividen en tres tipos en función de la realización sintáctica de los argumentos. Los de experimentante dativo y fuente en nominativo, como gustar, pueden tener una interpretación agentiva si la fuente es animada -El presidente solo dijo eso para gustar al electorado-; en tal caso, la subordinación a hacer se vuelve aceptable (28a-b) en tanto que Víctor hace algo para que las componentes del grupo Spice Girls hagan algo para gustar a Sara ${ }^{15}$

15 Nótese que (28a), frente a (27a), favorece la LOBL gracias a la anteposición del causee, en consonancia con lo argumentado por Torrego (2010). Sin embargo, esto se debe en realidad a que todos los argumentos de la oración son humanos, de modo que el orden no marcado agente (las Spice Girls) - paciente (Sara) facilita la lectura en la que ellas, las cantantes, hacen algo que afecte a Sara. 
o los miembros del Barça hacen algo para lograr gustar a la afición ${ }^{16}$. Si la fuente es inanimada (28c) o no se interpreta de manera agentiva (27a), no es posible interpretar la cadena causal correctamente.

(28) a. Víctor hizo a las Spice Girls gustar(le) a Sara.

b. [El Barça] quiere hacerse gustar y o bien acabará enamorando $[\ldots]^{17}$.

c. \#Víctor hizo a los donuts gustar(le) a Sara.

Los verbos psicológicos de experimentante sujeto, como odiar, no suponen un problema al hilo de esta discusión porque no cuentan con ninguna aplicativa, y son compatibles con las CCAA independientemente del hecho de que sean, por lo general, estativos y no agentivos - así, Sara provoca en Víctor un estado de odio hacia los sándwiches de Rodilla (29)_. Por su parte, los verbos de experimentante objeto, que tampoco introducen aplicativas, se dividen entre los que cuentan con un sujeto agentivo, como asustar, y los que cuentan con un sujeto no agentivo, como aburrir (Marín 2011). Los primeros son totalmente compatibles con las CCAA, al permitir una interpretación correcta de la cadena causal (30a) en la que Andrea causa que Diana haga algo para asustar a Sol (LCM), o bien la obliga a asustarla (LOBL). Con los segundos se puede forzar, así mismo, una lectura agentiva — y eventiva — similar a la de (28a), gracias precisamente a la intervención de un causante indirecto que instiga al causee — animado - a hacer algo volitivamente.

(29) Sara hizo a Víctor odiar los sándwiches de Rodilla.

(30) a. Andrea hizo a Diana asustar a Sol.

b. Antonio hizo a Cristina aburrir a los alumnos.

Por otro lado, las aplicativas altas que aparecen con verbos no psicológicos como ocurrir o suceder plantean un problema estructural para las CCAA:

16 La subordinación de estados a hacer no resulta sistemáticamente agramatical, pero sí poco aceptable e inusual. De acuerdo con Moreno Cabrera (2003), Morimoto (2011) y Carrasco Gutiérrez (2017), las situaciones controladas no pueden considerarse verdaderamente estativas. De ahí se sigue que las oraciones de (28) requieran la interpretación controlada y eventiva para resultar plenamente aceptables (véase nota anterior).

17 Ejemplo extraído de CREA, El País 09/01/1997: «El Barcelona se impone con claridad al Borussia en la ida de la Supercopa». 
(31) a. Cristina hizo ocurrir varias desgracias.

b. Debemos aprender a luchar por nuestros sueños y hacerlos suceder ${ }^{18}$.

c. *Cristina hizo ocurrir(le)/ sucederle varias desgracias a Antonio.

Ocurrir y suceder son verbos inacusativos que denotan un cambio, en principio espontáneo, compatible con la causación indirecta si se subordina a hacer (31a-b). Sin embargo, (31c) resulta agramatical por una cuestión de jerarquía sintáctica: el argumento introducido por una aplicativa alta, en este caso Antonio, ha de entrar en relación con todo el evento que domina (32a), lo cual es imposible si dicho evento viene causado por otro que aparece en una posición más alta, fuera de su dominio (32b). Es decir, Antonio puede recibir o poseer el evento ocurrir varias desgracias, pero no el evento complejo Cristina hacer ocurrir desgracias porque se encuentra entre hacer y ocurrir, en lugar de dominar a hacer.

(32) a. [SApl. DP Antonio [Apl.] [sv ocurrir varias desgracias]]

b. ${ }^{*}\left[_{\mathrm{SVO}_{\mathrm{V}}}\right.$ DP Cristina $[\mathrm{Voz}]\left[_{\mathrm{Sv}}\right.$ hacer [SApl. DP Antonio [Apl.] $\left[_{\mathrm{Sv}}\right.$ ocurrir varias desgracias]]]]

En definitiva, las anomalías que surgen al subordinar aplicativas altas a hacer pueden explicarse de manera independiente y no demuestran que el causee venga introducido por este tipo de núcleo.

Para terminar este apartado, se rechazan a continuación dos argumentos de Torrego (2010) a favor de un análisis con aplicativa para el español, y se presentan otros dos argumentos, además de los anteriores, en contra de tal análisis. En primer lugar, Torrego aduce que hacer marca con caso dativo el causee aun cuando este es el $\mathrm{AE}$ de un verbo inergativo:

(33) Juan ha hecho hablar *(a) una alumna.

(Torrego 2010: 455)

No obstante, es sabido que el español es una lengua con marcado diferencial de objeto, donde todos los CCDD definidos y animados reciben la misma marca - preposición $a$ - que los CCII (34). La pronominalización (35) demuestra que el sujeto del infinitivo en (33) tiene en realidad caso acusativo.

(34) a. Vi*(a) Nerea.

b. $\mathrm{Vi}(* a)$ la berenjena.

(35) Juan la ha hecho hablar.

$\overline{18}$ Ejemplo extraído de Google: http://hazlo-suceder.blogspot.com.es/ [última consulta: 24/04/2018]. 
En segundo lugar, Torrego señala que hacer no puede subordinar un verbo inacusativo (36a) a menos que se añada una aplicativa (36b), lo cual, en su opinión, demuestra que esta es requerida por el verbo ${ }^{19}$ :

(36) a. *Juan ha hecho llegar \{un / el paquete\}.

(Torrego 2010: 456)

b. Le ha hecho llegar un paquete con libros (a Luis).

Los ejemplos de (37) demuestran, sin embargo, que esta generalización es incorrecta, pues los inacusativos son aceptables en las CCAA sin necesidad de una aplicativa:

(37) a. El mago ha hecho desaparecer un conejo.

b. El jardinero hizo florecer la camelia empleando un nuevo fertilizante.

c. Andrea hizo salir agua del pozo.

En tercer lugar, el AE del infinitivo en español, como en el resto de las lenguas romances, puede recibir acusativo si no compite con un AI (38). Esto resulta muy difícil de explicar si viene introducido por un núcleo aplicativo, como señalan también Folli y Harley (2007), ya que este asigna dativo invariablemente (cfr. Cuervo 2003; Pylkkänen 2008).

(38) Nerea la hizo llorar.

En cuarto lugar, las CCAA son recursivas, lo cual quiere decir que la proyección de hacer puede subordinar otra idéntica ilimitadamente. Asumiendo que el AE de hacer — Marta en (39) - es introducido por Voz, el primer verbo hacer habría de estar subordinando una estructura no idéntica a la suya propia si el AE del infinitivo -Patricia - se encontrara en una aplicativa:

(39) a. Marta hizo a Patricia hacer llegar la carta.

b. [SVoz Marta [Sv hizo [Apl. a Patricia][Sv hacer [SV llegar la carta]]]]

En conclusión, si las oraciones recursivas como las de (39) se forman al subordinar segmentos idénticos de estructura, el AE del infinitivo ha de ser introducido por $\mathrm{Voz}^{20}$, igual que el de hacer. Como se explicará a continuación, este análisis del causee tiene dos consecuencias para la sintaxis de las CCAA: en primer lugar, el

19 Esta aplicativa sería, por otra parte, una aplicativa baja que introduce un destinatario.

20 Wurmbrand (2015b) defiende también un análisis SVoz para otro tipo de verbos de reestructuración. 
infinitivo no puede encontrarse en un SV escueto y, en segundo lugar, para explicar el orden de palabras es necesario asumir que es el verbo el que se desplaza a una posición por encima de su AE.

\section{EL TAMAÑO DEL INFINITIVO}

Establecer la estructura funcional del infinitivo es un problema crucial para dilucidar la relación que guarda con hacer en las CCAA, es decir, para comprender cómo se ensamblan sintácticamente las dos partes del PC. A lo largo de los años se ha debatido sobre la presencia de proyecciones como ST o SC en el infinitivo ( $c f r$. Baker 1988), sobre si este y hacer se encuentran o no dominados por un mismo núcleo $\mathrm{V}$ (cfr. Geach 1970; Kayne 1975; Alarcos 1970; Hernanz 1982; Moreno Cabrera 1987; Fernández Lagunilla \& Dios López 1991; Treviño 1994; Butt 1995; Meurers 2000; Saito \& Hoshi 2000; Williams 2003) ${ }^{21}$ y sobre si el infinitivo se encuentra o no en un SV escueto (cfr. Wurmbrand 2001; Folli \& Harley 2007; López 2001; Torrego 2010). En este apartado se confirmará la hipótesis de que las CCAA del español son estructuras monoclausales que preservan dos SSVV, revisando los argumentos tradicionales y añadiendo otros nuevos. Se defenderá además que la proyección inferior —la del infinitivo - es defectiva y llega solo hasta SAsp.

Se comenzará por desgranar las propiedades de las CCAA como PPCC de reestructuración, es decir, aquellas que evidencian que hacer y el infinitivo conforman un único dominio sintáctico con un solo ST, un solo SM-epistémico y un solo entorno para la asignación de caso y para el movimiento de clíticos. Concretamente, los datos aportados por los modales contribuirán significativamente a cartografiar la estructura de las CCAA, pues se observará que el infinitivo puede albergar una proyección SM-deóntico. A continuación, se demostrará que este alberga también SAsp, posición a la que se desplaza — en busca de la desinencia $-r$ - dando lugar al orden de palabras no marcado. Finalmente, se revisarán aquellas propiedades de las CCAA que demuestran que hacer y el infinitivo conservan cierto grado de independencia y se encuentran en SSVV distintos.

\subsection{Asignación de caso}

Las CCAA constituyen un único dominio para la asignación de caso, de modo que la estructura argumental del PC es la suma del AE de hacer y todos los argumentos del

21 En la tradición hispánica, el análisis de hacer y su infinitivo como una unidad se encuentra ya en Bello (1847). 
infinitivo. La presencia de un solo nominativo, reservado al $\mathrm{AE}$ del verbo flexionado, apunta a la presencia de un único núcleo T. El causee, en cambio, recibe un caso por defecto: acusativo si no compite con un AI (40), dativo en caso contrario (41).

(40) a. Nerea la hizo llorar (a su enemiga).

b. Nerea las hizo desaparecer (las pruebas).

(41) a. Patricia le hizo ver cine clásico (a Marta).

b. Patricia le hizo (a Marta) darle el libro a Nerea.

Si bien los ejemplos de (40) pueden dar pie a analizar el causee como CD de hacer o incluso del conjunto hacer + infinitivo (cfr. RAE 1973; Alsina 1992; Guasti 1996; Ciutescu 201322), los ejemplos de (41) demuestran que tal análisis es insostenible, ya que este argumento recibe un caso por defecto que puede variar y que no le asigna directamente ningún núcleo verbal ${ }^{23}$.

En este artículo introducimos una hipótesis de trabajo sobre la proyección $\mathrm{S} \alpha$ (inspirada en López 2012), responsable de la asignación de acusativo y, en última instancia, de la presencia / ausencia de SVoz. S $\alpha$, ubicado entre Sv y SAsp, tiene dos valores en distribución complementaria que se corresponden grosso modo con los «sabores» (flavours) o rasgos de $v$ en Folli y Harley (2004) o Cuervo (2003): $\alpha\{+\mathrm{t}($ ransitivo)\} coteja acusativo y legitima la inserción de Voz, mientras que $\alpha\{$-t(ransitivo)\} no coteja caso y, en consecuencia, no permite la inserción de Voz, dando así lugar a estructuras inacusativas.

La principal motivación para postular este núcleo funcional estriba en que la información concerniente a la inserción del AE debe separarse de v, pues este funciona solo como un núcleo verbalizador (Vivanco 2016): aquellos modelos que asumen que el «sabor» de $\mathrm{v}$ es lo que determina la presencia $\left(\mathrm{v}_{\mathrm{DO}}, \mathrm{v}_{\mathrm{CAUSE}}\right)$ o ausencia $\left(\mathrm{v}_{\mathrm{BECOME}}\right)$ de Voz topan con un problema a la hora de explicar por qué la morfología puramente

22 Alsina (1992) defiende que fare en italiano tiene tres argumentos: agente, evento y paciente, y que este último se fusiona con un argumento del infinitivo. Guasti (1996) propone que el causee recibe caso del PC una vez que el infinitivo se incorpora a hacer. Por su parte, Ciutescu (2013) propone dos análisis distintos para las CCAA: uno de reestructuración para las CCAA que presentan el causee en posición posverbal y otro de MDO para las que presentan un causee antepuesto. Basándose en ejemplos del tipo La hizo leer el documento, Ciutescu (2013: 49) defiende que los causees preverbales reciben acusativo cuando se pronominalizan; sin embargo, el ejemplo en cuestión se trata de un laísmo y, además, no es posible saber si el clítico la se corresponde con un causee pre- o posverbal en su origen.

23 Además, señala Hernanz (1999) que, si el sujeto acusativo fuera el CD de hacer, tendería a aparecer adyacente a él en el orden no marcado, y no tras el infinitivo. 
verbalizadora - cuya posición ha de ser v- y la morfología (anti)causativa pueden coaparecer (Key 2013):

(42) Cristina se $\mathbf{e}_{\text {morf. anticausativa }}$ puso $_{\text {verbo ligero }}$ nerviosa.

Key resuelve este problema proponiendo, dentro del marco de la Morfología Distribuida, que v sufre una operación postsintáctica de fisión, tal que sus rasgos verbalizadores se separan de sus rasgos cause / become. No obstante, la fisión fue originalmente concebida para dar cuenta de aquellos casos en los que dos o más exponentes materializan un solo rasgo (Noyer 1997), y no para casos, como el de (42), en los que dos exponentes distintos materializan dos rasgos distintos. Por ello, de acuerdo con lo argumentado en Vivanco $(2016)^{24}$, resulta más plausible atribuir a $v$ una función meramente verbalizadora, ubicando la información relativa a la inserción del AE en un núcleo distinto, asociado al caso.

De acuerdo con Marantz (1991), la materialización concreta del caso morfológico en la interfaz fonológica no ha de corresponderse necesariamente con el caso abstracto que legitima los SSNN en la derivación. Esto tiene dos consecuencias básicas. En primer lugar, un argumento recibe acusativo no solo por sus propiedades configuraciones o argumentales, sino en oposición a otro argumento. En segundo lugar, el caso puede ser asignado por defecto, en función del contexto sintáctico, cuando otros mecanismos de asignación fallan.

De este modo, si $\alpha$ tiene el rasgo $\{+t\}$ asigna caso al AI, pero el AE no puede desplazarse a $\mathrm{T}$, por lo que debe obtener caso del primer núcleo que le manda-c: el $\alpha\{+\mathrm{t}\}$ de hacer. La realización morfológica de los tres casos se produce, siguiendo a Marantz, por competición: el sujeto de hacer y el AI del infinitivo se materializan como nominativo y acusativo de manera regular, mientras que el causee se materializa como dativo por defecto, al perder la competición por el acusativo frente al AI.

(43) Bowie hizo a Víctor cantar Space Oddity $\rightarrow$ se la hizo cantar.

(44) $\left[_{\mathrm{ST}} \mathrm{SN}_{\mathrm{NOM}}\right.$ Bowie $_{\mathrm{i}}[\mathrm{T}]\left[_{\mathrm{SVoz}} h_{\mathrm{i}}[\mathrm{Voz}]\left[_{\mathrm{Sv}}[\mathrm{v}\right.\right.$ hacer $]\left[_{\mathrm{S} \alpha}\left[\alpha_{\{+t\}}\right]\left[_{\mathrm{SVoz}} \mathbf{S N}_{\mathrm{DAT}}\right.\right.$ Victor $[\mathrm{Voz}]$ $\left[{ }_{\mathrm{Sv}}[\mathrm{v}\right.$ cantar $]\left[\left[_{\mathrm{S} \alpha}\left[\alpha_{\{+\mathrm{t}\}}\right]\left[_{\mathrm{Sv}}[\mathrm{V}]\left[\mathrm{SD}_{\mathrm{ACU}}\right.\right.\right.\right.$ Space Oddity $\left.\left.\left.\left.\left.\left.\left.\left.]\right]\right]\right]\right]\right]\right]\right]\right]$

En ausencia de un AI, en cambio, el causee puede materializarse como acusativo porque no compite con otro argumento por este caso ${ }^{25}$ :

$24 \quad$ Véase Vivanco (2016) para la discusión de otras posibles soluciones a este problema.

25 Nótese que el $\alpha\{+\mathrm{t}\}$ del infinitivo no tiene, en esta ocasión, ningún argumento al que asignar caso. El núcleo $\alpha$ es $\{+\mathrm{t}\}$ en presencia de un AI; esto es lo que permite la inserción de Voz: si $\alpha$ es 
(45) Nerea hizo llorar a su enemiga $\rightarrow$ la hizo llorar.

(46) $\left[_{\mathrm{ST}}\left[\mathrm{SD}_{\mathrm{NOM}}\right.\right.$ Nerea $_{\mathrm{i}}[\mathrm{T}]\left[_{\mathrm{SVoz}} h_{\mathrm{i}}[\mathrm{Voz}]\right]_{\mathrm{Sv}}[\mathrm{v}$ hacer $]\left[_{\mathrm{S} \alpha}\left[\alpha_{\{+\mathrm{t}\}}\right]\left[_{\mathrm{SVOz}} \mathrm{SD}_{\mathrm{ACU}} s u\right.\right.$ enemiga $[\mathrm{Voz}]\left[_{\mathrm{Sv}}[\mathrm{v}\right.$ llorar $\left.\left.\left.\left.\left.]\left[\left[_{\mathrm{S} \alpha}\left[\alpha_{\{+\mathrm{t}\}}\right]\left[_{\mathrm{SV}}[\mathrm{V}]\right]\right]\right]\right]\right]\right]\right]\right]$

Finalmente, si $\alpha$ es $\{-\mathrm{t}\}$, el infinitivo es inacusativo: el AI no recibe caso y Voz no se proyecta. De este modo, el AI no puede desplazarse a $\mathrm{T}$ para recibir nominativo, así que recibe caso del $\alpha\{+\mathrm{t}\}$ de hacer. Dicho caso se materializa como acusativo porque no hay competencia con otro SD:

(47) Nerea hizo desaparecer las pruebas $\rightarrow$ Las hizo desaparecer.

(48) $\left[_{\mathrm{ST}} \mathrm{SD}_{\mathrm{NOM}}\right.$ Nerea $_{\mathrm{i}}[\mathrm{T}]\left[_{\mathrm{SVoz}} h_{\mathrm{i}}[\mathrm{Voz}]\left[_{\mathrm{Sv}}[\mathrm{v}\right.\right.$ hacer $]\left[_{\mathrm{S} \alpha}\left[\alpha_{\left\{+\mathrm{t}_{\}}\right.}\right]\left[_{\mathrm{Sv}}[\mathrm{v}\right.\right.$ desaparecer $]$ $\left[{ }_{\mathrm{S} \alpha}\left[\alpha_{\{-\mathrm{t}\}}\right]\left[\mathrm{SV}[\mathrm{V}]\left[\mathrm{SD}_{\mathrm{ACU}}\right.\right.\right.$ las pruebas $\left.\left.\left.\left.\left.\left.\left.]\right]\right]\right]\right]\right]\right]\right]$

En resumen, los SSDD reciben caso en función de una jerarquía nominativo $>$ acusativo $>$ dativo: si solo hay dos SSDD, recibirán nominativo y acusativo, independiente de su posición y de su rol temático; si hay tres SSDD, aquel con el que fallen los mecanismos habituales de asignación de caso recibirá dativo por defecto.

Para concluir este apartado, cabe señalar un contraste más. En Vivanco (2016) los exponentes se у $\varnothing$ compiten para materializar el núcleo $\alpha\{-t\}$ en contextos anticausativos — la ropa se secó frente a mi vida cambió-Ø_. Ahora bien, en español la presencia del se anticausativo es aparentemente opcional en las CCAA:

(49) a. El sol hizo secar-Ø la ropa.

b. El sol hizo secarse la ropa.

Estas construcciones son poco habituales en castellano, pues se prefiere la variante con SC el sol hizo que la ropa se secara, y han sido poco tratadas en la bibliografía, con la excepción de los trabajos de autoras como Zubizarreta (1985), para quien el clítico se no es opcional, sino obligatorio, en estos contextos; Folli y Harley (2007), quienes señalan que si es incompatible con fare en italiano; y Cuervo (2016), que describe la variación entre la presencia / ausencia de se en las CCAA y trata de dilucidar los factores que la determinan.

$\{-\mathrm{t}\}$, no hay acusativo y no se permite la inserción de un argumento extra vía Voz. En el caso de los inergativos asumimos, siguiendo a Hale y Keyser (2002), que cuentan en realidad con un AI incorporado al verbo, que satisface el rasgo de caso de $\alpha$. 
De acuerdo con lo expuesto en este apartado, puede parecer, a priori, que la materialización expresa de $\alpha\{-t\}$ resulta superflua en (49): $\alpha\{-t\}$ se caracteriza por no asignar acusativo al AI e impedir la inserción de un AE; sin embargo, en una CA encontramos ambas cosas, el AE de hacer y el caso acusativo asignado al AI del infinitivo por el $\alpha$ de hacer. No obstante, el problema es en realidad más complejo. Vivanco (2016) argumenta que el aspecto, a su vez determinado por la estructura escalar inherente a los verbos de cambio de estado, es uno de los factores decisivos en la competición entre se y Ø por materializar $\alpha$ : se aparece cuando la escala tiene dos o más grados, está cerrada y da lugar a un estado resultante ${ }^{26}$. Si no se dan estas condiciones, se puede estar ausente incluso con aquellos verbos con los que es supuestamente obligatorio (secar, enfriar):

(50) Puse la comida a calentar(*se).

(Vivanco 2016: 228)

(51) a. Dejé secar la ropa.

(Vivanco 2016: 229)

b. Dejé secarse la ropa.

En la línea de Labelle (1992), Vivanco explica que la perífrasis poner algo a selecciona predicados atélicos, lo que obliga a la ausencia de se. Por otra parte, (51) presenta un contraste aspectual: (51a) se interpreta como «yo dejé que el proceso de secado comenzara, pero no me preocupé necesariamente de si concluyó o no», mientras que (51b) se interpreta como «controlé el proceso hasta su culminación». Esta misma diferencia puede observarse en (49a) frente a (49b). Sin embargo, no es posible, por el momento, afirmar que el aspecto sea la única clave para comprender la variación. El problema de la subordinación de anticausativas en causativas analíticas de distinta índole - dejar es un verbo causativo de reestructuración, como hacer, y la perífrasis poner algo a tiene así mismo un valor causativo - requiere un estudio mucho más profundo de lo que permiten los límites de este trabajo, por lo que aquí queda tan solo planteado para futuras investigaciones.

\subsection{Clíticos}

Es sabido que los clíticos se distribuyen en las CCAA como en una oración simple, en torno al complejo <hacer + infinitivo>, y que ascienden para apoyarse en el verbo flexionado como si de una sola unidad se tratase (Kayne 1975).

26 Vivanco (2016) distingue entre logros simples (no escalares) y complejos (escalas de dos grados) de cambio de estado, además de las escalas múltiples que dan lugar a interpretaciones de actividad (abiertas) o realización (cerradas). 
(52) a. Nerea (la) hizo $(* l a)$ llorar $(* l a)$.

b. El profesor (les) hizo (*les) leer(*les) Pedro Páramo.

c. El profesor se lo hizo leer.

d. El profesor les hizo leerlo.

e. El profesor (lo) hizo leer(lo) a los alumnos.

Solo el CD del infinitivo es libre de elegir entre pronominalizarse sobre este, con el que forma un constituyente, o ascender al verbo flexionado (52c-e), mientras que el causee debe apoyarse obligatoriamente sobre este último. Por su parte, el AI de los infinitivos inacusativos (53) no se comporta a este respecto como un objeto, sino como un sujeto. La razón es que el $\alpha\{-\mathrm{t}\}$ del infinitivo no le asigna caso: en circunstancias normales, el AI se desplazaría al especificador de T para obtener nominativo pero este se encuentra ocupado por el AE de hacer, de modo que solo puede recibir caso del $\alpha\{+\mathrm{t}\}$ de hacer $\mathrm{y}$, por tanto, solo puede pronominalizarse sobre él.

(53) Nerea (las) hizo desparecer(*las) (las pruebas).

El ascenso de clíticos es un diagnóstico estándar de reestructuración ( $c f r$. Kayne 1975; Aissen \& Perlmutter 1983; Wurmbrand 2001, entre otros muchos), pues evidencia que no hay una barrera oracional entre hacer y el infinitivo, a diferencia de lo que sucede con los infinitivos sin reestructuración del tipo *la odio ver. Recordemos que en el ejemplo (14) —* La hizo a Leonardo construir (Treviño 1992: 316) — vimos que la anteposición del causee bloquea el ascenso del clítico de objeto, lo cual podría interpretarse como un argumento en contra de un análisis de reestructuración para las CCAA o, al menos, para las CCAA en su versión con causee preverbal (Ciutescu 2013, 2015). Sin embargo, considero que este efecto de intervención no repercute en la estructura general de las CCAA, las cuales constituyen un único domino para la asignación de caso independientemente de la posición del causee; más bien, se trata de una evidencia adicional de que hacer y el infinitivo preservan en español un grado de independencia mayor que en otras lenguas, sin dejar por ello de constituir un $\mathrm{PC}^{27}$ (véase el apartado 3.6).

Kayne (1975) utiliza otros fenómenos relacionados con los clíticos para demostrar que el infinitivo y hacer no están dominados por un mismo núcleo $\mathrm{V}$, aunque la estructura sea monoclausal. Esto es lo esperable si tenemos reestructuración, en lugar de incorporación. Así, en primer lugar, los clíticos de sujeto han de colocarse en francés a la derecha del verbo en las interrogativas, y en el caso de las CCAA se

Ciutescu (2013) analiza las CCAA con causee antepuesto como construcciones de MEC. 
colocan a la derecha de hacer, no del infinitivo (54); es decir, el sujeto de hacer le «pertenece» en exclusiva ${ }^{28}$. Por su parte, los clíticos de objeto también se colocan a la derecha del verbo en el imperativo — tanto en francés como en español_- y eligen hacer, en lugar del infinitivo, en las causativas (55). En definitiva, hacer y el infinitivo se encuentran en posiciones distintas - lo que permite intercalar los clíticos de estos ejemplos — dentro de una estructura monoclausal sin barreras que impidan el ascenso.

(54) Fera-t- $(i l)$ partir- $(* i l)$ Marie?

(Kayne 1975: 217)

hará-él marchar-él María.

(55) Ház(le) leer(*le) ese libro.

\subsection{La pasivización y los modificadores temporales: un único ST}

La pasiva a larga distancia (long passive) es otro diagnóstico clásico de reestructuración (Hernanz 1982; Wurmbrand 2001, 2004) y, además, un diagnóstico para identificar verbos léxicos de reestructuración — frente a los funcionales (Cinque 2001) - . En (56-57) vemos que el italiano y el español permiten pasivizar el objeto del infinitivo ${ }^{29}$, frente al francés (58):

(56) a. Il pachetto fu fatto arrivare (da Gianni).

(F\&H 2007: 225) el paquete fue hecho llegar (por Gianni)

b. Il libro fu fatto leggere a Mario (da Gianni). el libro fue hecho leer a Mario (por Gianni)

(57) a. El ofrecimiento fue hecho llegar al ministro, Jaime Mayor Oreja $[\ldots]^{30}$.

b. [...] [él] fue hecho callar por el capellán, que no pudo entender aquella intromisión luterana dentro de su iglesia ${ }^{31}$.

$28 \quad$ En español (glosas) este contraste no se aprecia porque no existen clíticos de sujeto, y los pronombres tienen mayor libertad posicional.

29 La pasivización del AE del infinitivo resulta agramatical en las lenguas discutidas (cfr. Folli \& Harley 2007), como es esperable, pues no es el complemento directo de hacer, o del PC: *El niño fue hecho llorar (por Nerea). Como señala un revisor anónimo, sí son gramaticales en español construcciones del tipo Pepe se hace querer por todo el mundo, donde el CD del infinitivo es correferencial con el sujeto de hacer (reflexividad) y donde la interpretación, a juzgar por la realización del causee como un complemento agente con por, es pasiva. Estas construcciones requieren un estudio más profundo que excede los límites de esta investigación.

30 CREA, Canarias 7, 25/05/1999.

31 CREA, García Márquez, Gabriel (1985 [1987]): El amor en los tiempos del cólera, Madrid: Mondadori. 
(58) *Le livre a été fait lire (à Margot). el libro ha sido hecho leer a Margot

En consonancia con lo apuntado por Folli y Harley (2007) y Campanini y Pitteroff (2013), este contraste sugiere que faire tiene un estatus más funcional que fare ${ }^{32}$, lo cual le impide formar pasivas ${ }^{33}$. Así pues, siguiendo con la argumentación de la sección 2.1.2., hacer tiene en español un estatus más cercano al de un verbo léxico pleno que al de un elemento funcional, lo cual explica esta y otras peculiaridades de su comportamiento sintáctico, como su capacidad para subordinar un SC.

Por otra parte, las tres lenguas coinciden al rechazar la subordinación de pasivas en el infinitivo:

(59) a. *Diana hizo ser invitada a Andrea.

b. *Jean fera être invitée Margot. Jean hará ser invitada Margot.

c. *Giovanni farà essere invitato Piero. Giovanni hará ser invitado Piero.

Esto se debe a que los PPCC son construcciones monoclausales: si el especificador del único ST está ocupado por el AE de hacer, ningún argumento del infinitivo puede ascender a él y, por lo tanto, no puede pasivizar recibiendo nominativo y convirtiéndose en sujeto sintáctico.

Por otra parte, es un hecho a menudo destacado en la bibliografía que los eventos causante y causado de las CCAA no han de coincidir necesariamente en el tiempo y que admiten modificadores temporales independientes (Fodor 1970):

(60) Víctor hizo el martes cantar a Sara el jueves.

La gramaticalidad de (60) evidencia que las CCAA se componen de dos SSVV, que proporcionan dos posiciones para insertar modificadores. No obstante, la información aportada por estos ha de ser coherente con aquella proporcionada por un único núcleo $\mathrm{T}$ :

32 Campanini y Pitteroff (2013) citan a Guasti (1993), quien señala que la pasivización de faire era gramatical en francés antiguo, y ven este dato como una prueba de la progresiva gramaticalización de faire.

33 Esto contradice a autores como Zubizarreta (1985) o Soares da Silva (2012), para quienes fare es más funcional que faire. Concretamente, Folli y Harley (2007) tratan fare como un elemento que puede ser funcional o léxico en italiano, dependiendo de si aparece en una CCAA de infinitivo o en las llamadas «faire-par causatives» del tipo: Gianni ha fatto riparare la macchina da Mario ('Gianni ha hecho arreglar el coche por Mario'), donde el causee es un complemento agente. 
(61) Víctor hizo el jueves pasado enfermar a Sara ayer.

$$
\text { Pasado + Pasado }
$$

\#Pasado + Futuro

(62) \#Víctor hizo ayer enfermar a Sara pasado mañana.

El evento causante y el causado pueden ser independientes temporalmente en las CCAA precisamente porque cada evento está materializado por un elemento distinto (61). En cambio, la oración de (62) resulta anómala pese a cumplir la condición de que el evento causante sea anterior al causado. Al comparar ambos ejemplos, se observa que en el primer caso los dos eventos tienen lugar en el pasado respecto al momento de habla, mientras que en el segundo, uno tiene lugar en el pasado y otro en el futuro, lo que resulta inaceptable dado que la falta de simultaneidad solo es posible siempre y cuando se respete la información del único ST: el infinitivo carece de información temporal y la toma del verbo flexionado o, en otras palabras, ambos verbos reciben la información de un mismo ST.

\subsection{La modalidad}

Los modales deónticos se encuentran justo encima del SV (root modals), mientras que los epistémicos tienen un ámbito mayor ( $c f r$. Jackendoff 1972; Picallo 1990; Brennan 1993; Cinque 1999; Hacquard 2006, 2009; RAE 2009). Así, si las CCAA son estructuras monoclausales con dos SSVV, se espera que haya una única posición para los modales epistémicos, y dos para los deónticos, predicción que confirman los datos presentados a continuación.

Un modal a la izquierda de hacer siempre puede interpretarse como epistémico (63a-b). La interpretación deóntica también está disponible en la LOBL (63a), pero queda bloqueada en la LCM (63b) por razones semánticas - resulta difícil interpretar la modalidad deóntica sobre una causa-. Es decir, a la izquierda de hacer hay tanto una posición alta para los modales epistémicos como una baja — sobre el SV—para los deónticos:

(63) a. Víctor debe [EPIST. / DEONT.] $_{\text {hacer cantar a Sara. }}$

b. El viento debió

En cambio, hacer no puede subordinar un modal epistémico, aunque sí uno deóntico (64). En este último caso, el deóntico bloquea aparentemente la LOBL, de nuevo por razones semánticas: resulta más difícil, aunque no imposible, concebir la modalidad deóntica como el resultado de la voluntad de un agente externo. 
(64) a. Víctor hizo a Sara poder ${ }_{\left[{ }^{* E P I S T} \text { / DEONT] }(\# \text { LOBL / LCM })\right.}$ cantar.

b. El buen tiempo hizo poder ${ }_{\left[{ }^{*} \text { EPIST. } / \text { DEONT.] }\right.}$ aterrizar el avión.

c. La presentación de esta subzona nos hizo poder catar en primeur los vinos de 27 de los 44 productores acogidos a esta denominación ${ }^{34}$.

Una última observación respecto a los deónticos es que favorecen la anteposición del causee (65) ${ }^{35}$. Como apuntamos en el apartado 2.1.2. (ejemplo 12), esto se debe a la necesidad de facilitar el procesamiento de una secuencia con tres verbos:

(65) a. Bowie / la necesidad hizo (a Víctor) deber cantar en el metro (?a Víctor).

b. El hambre hizo (a Víctor) tener que robar en el mercado (?a Víctor).

Estos datos permiten comenzar a esbozar el análisis sintáctico de las CCAA. Como vemos en (67), los modales epistémicos encuentran una única posición en las CCAA, igual que ST. Los deónticos, en cambio, encuentran dos posiciones en las que insertarse. Esto implica no solo que ambos verbos permanezcan en SSVV separados, sino que la estructura funcional que proyecta el infinitivo debe llegar al menos hasta SM-deóntico, o más allá.

(66) La operación debió ${ }_{\text {[EPIST./ DEONT.] }}$ hacer poder ${ }_{\text {[DEONT.] }}$ mejorar a Sara.

(67) [ST [SM-espist. [SAsp [SM-deont. [SV hacer [SAsp [SM-deont. [SV]]]]]]

\subsection{Aspecto gramatical}

En el apartado 3.3. se comprobó que los modificadores temporales encuentran dos posiciones en las CCAA, en cada uno de los SSVV, aunque solo hay un ST aportando información temporal. La situación es diferente en relación con el aspecto, pues cada evento puede tener una información aspectual distinta: el causado puede ser imperfectivo aun cuando el causante es aoristo (68), y viceversa (69):

$$
\text { Aoristo }+ \text { imperfecto } \rightarrow \text { aoristo }
$$

(68) En diez minutos, Víctor hizo a Sara estar tocando el teclado (una hora / \#ahora).

$$
\text { Imperfecto }+ \text { aoristo } \rightarrow \text { imperfecto }
$$

(69) May está haciendo a Gianni terminar la traducción en diez minutos.

\footnotetext{
34 Ejemplo extraído de CREA, El Mundo, 03/01/2003.

35 Con animados, ya que los inanimados parecen rechazar la anteposición.
} 
Estos ejemplos apuntan, por tanto, a la presencia de un SAsp sobre el infinitivo, capaz no solo de albergar modificadores, sino de aportar su propia información aspectual. Nótese, no obstante, que la interpretación final de (68) es de aoristo, mientras que la de (69) es imperfectiva. Esto significa que la especificación aspectual de hacer es la que domina la oración y que, por tanto, el SAsp del infinitivo no se encuentra en un dominio oracional independiente.

Por esta razón, la repetición de la misma información aspectual en los dos eventos - imperfecto en (70a) y perfecto en (70b) - resulta redundante y anómala:

(70) a. ?Víctor está haciendo a Sara estar tocando el teclado (ahora mismo).

b. ?May hizo en diez minutos terminar la traducción a Gianni en media hora.

Cabe señalar que la agramaticalidad de ejemplos como los de (71) no se debe a cuestiones aspectuales como las que venimos tratando en este apartado, sino a la violación de la condición semántica según la cual el evento causante ha de ser anterior al causado.

(71) a. *May ya ha hecho haber terminado la traducción a Gianni.

b. *May está haciendo ya haber terminado la traducción a Gianni.

Asumimos, siguiendo a autores como Felser (1999), que el especificador de SAsp genera un argumento eventivo - nótese que la subordinación de estados a hacer es infrecuente y anómala: \#Escuchar a Mozart hizo a la niña ser inteligente- - y que, en circunstancias normales, tal argumento recibe una identificación temporal mediante la concordancia con T; es decir, el tiempo de foco ha de ponerse en relación con el tiempo de la situación. En cambio, en estructuras subordinadas sin ST como las CCAA, el argumento eventivo de SAsp puede ponerse en relación con el T de hacer a través de una relación de control ${ }^{36}$. Esto explicaría de manera natural datos como los de (68-69) en los que cada sub-evento puede tener un aspecto gramatical distinto al tiempo que el evento complejo recibe en última instancia la interpretación aspectual del subevento más alto, en relación con el único ST disponible en la estructura.

En conclusión, SAsp es la proyección funcional más alta del infinitivo, a la que este se desplaza para incorporarse a la desinencia - $r$. Así, esperamos que el infinitivo cuente también con todas las proyecciones funcionales que se encuentren por debajo de SAsp — SM-deóntico, SVoz —, pero ninguna por encima —SM-epistémico, ST—.

36 Véase Carrasco Gutiérrez (2017) para una revisión crítca de esta propuesta en relación con los verbos de percepción. 


\subsection{La independencia de hacer y el infinitivo}

La asignación de caso, la distribución de los clíticos y la supuesta inseparabilidad de hacer y el infinitivo son algunos de los factores que han favorecido diversos análisis de hacer y el infinitivo como una unidad, o incluso como una perífrasis. Sin embargo, en apartados anteriores han aparecido ejemplos en los que la secuencia formada por ambos verbos se ve interrumpida por el causee (72a) - a diferencia, recordemos, del francés y el italiano-, un clítico (72b), o un modal deóntico (72c):

(72) a. Víctor hizo a Sara cantar hasta quedarse afónica.

b. Hazme reír.

c. El prólogo me hizo poder entender mejor a Proust.

Además, hacer y el infinitivo pueden ser elididos de manera independiente (Kayne 1975), lo que demuestra que no forman un constituyente:

(73) a. Luis hará hablar a Macarena y callar a Yuridia.

b. Cora ha hecho beber y bailar a Luisa.

Kayne (1975: 263) también apunta que el ligamiento de los recíprocos no traspasa en las CCAA la frontera entre hacer y el infinitivo, lo cual indica que no están dominados por un mismo $\mathrm{V}$ :

(74) a. *Ellos han hecho a la rectora discutir los unos con los otros. (i.e. que discuta con unos y con otros).

b. *Ellos les hicieron a los manifestantes lanzar piedras el uno sobre el otro.

Por otra parte, Bordelois (1988) señala que no es posible interpolar la negación o un auxiliar entre hacer y el infinitivo (75), lo cual indica, en su opinión y en la de otros autores como Campanini y Pitteroff (2013), que no hay ninguna proyección funcional en el complemento infinitivo o que, de haberla, es defectiva:

(75) a. *Él lo hizo no venir.

b. *Él lo hizo haber venido.

(Bordelois 1988: 60)

Sin embargo, estos datos no son correctos. La agramaticalidad de (75b) se debe a la violación de la condición de que el evento causado sea posterior al causante, y la de (75a) no es tal, como demuestran los siguientes ejemplos extraídos del CREA: 
(76) a. [...] una lesión le hizo no lucir al ciento por ciento ${ }^{37}$.

b. Una fe en la que me han hecho no creer $^{38}$.

c. Mi escepticismo respecto a cualquier fenómeno parapsicológico me hizo no prestar atención a la indicación premonitoria $[\ldots]^{39}$.

Esto supone no solo que hacer y el infinitivo no están dominados por un mismo núcleo $\mathrm{V}$ - no hay incorporación-, sino que este último ha de contar con alguna proyección funcional para albergar la negación ( $c f r$. Torrego 2010), a pesar de carecer de ST. Recordemos, además, el ejemplo (14) — * La hizo a Leonardo construir (Treviño 1992: 316) —, que demuestra que el ascenso de clíticos puede verse bloqueado en determinados contextos y que, por lo tanto, la unión entre los dos verbos no es absoluta.

En definitiva, confirmamos y asumimos la idea clásica de que las CCAA son estructuras monoclausales, lo cual no implica que hacer y el infinitivo funcionen a todos los respectos como un solo verbo. La estructura preserva dos SSVV hasta cierto punto independientes, pues hacer y el infinitivo pueden ser interrumpidos por otros elementos, pueden ser elididos de manera independiente y ejercen su propio dominio para el ligamiento. Además, el comportamiento de la negación y la posibilidad de anteponer el causee apuntan a que entre ellos hay más estructura funcional de la que suele presuponerse en la mayoría de los análisis.

\section{PROPUESTA DE ANÁLISIS}

Dentro de la gramática generativa, los trabajos que han abordado el tema de las causativas analíticas y sintéticas asumen que una estructura biclausal queda reducida en otra monoclausal. La diferencia entre las distintas propuestas radica en la implementación de tal reducción, especialmente respecto al módulo de la gramática en que tiene lugar.

En Gramática Transformacional, dicho fenómeno se entendía como una transformación desde la estructura profunda a la estructura superficial (cfr. Inove 1969; Kuroda 1965; Kuno 1973; Shibatani 1973; Hernanz 1982). Después, los modelos lexicistas defendieron que las operaciones que determinan la complejidad morfológica y semántica de una forma simple han de producirse en el propio léxico, lo cual es especialmente relevante para las causativas sintéticas, creadas mediante un proceso de sufijación (cfr. Farmer 1980; Miyagawa 1980; Grimshaw \& Mester 1985; Butt 1995; Manning

\footnotetext{
$37 \quad$ CREA, Diario de Yucatán, 09/09/1996.

38 CREA, Marín Recuerda, José (1980 [1991]): Las arrecogías del beaterio de Santa María Egipcíaca, Madrid: Cátedra.

39 CREA, Boadella, Albert (2001): Memorias de un bufón, Madrid: Espasa Calpe.
} 
et alii 1999). Finalmente, los modelos construccionistas, entre los que se inscribe este estudio, identifican la complejidad semántica y la sintáctica en el árbol ( $c f r$. Folli \& Harley 2007), es decir, descomponen en la sintaxis los significados complejos de las expresiones lingüísticas, tanto si estas son morfológicamente simples como si no.

Como quedó apuntado en la introducción, este trabajo parte de la hipótesis de que hacer, como verbo de reestructuración, subordina una estructura oracional incompleta, siendo esto lo que le permite formar un PC con el infinitivo ( $c f r$. Wurmbrand 2007; Tubino Blanco et alii 2014). En otras palabras, no se produce una reducción en el léxico que envíe a la derivación una unidad ya simplificada, sino que la estructura compleja se preserva en la sintaxis (cfr. Baker 1988). Puesto que el infinitivo no proyecta en ningún momento ST o SC, la estructura comienza como monoclausal en la derivación y, en consecuencia, no hay necesidad de ninguna operación específica de reducción ( $c f r$. Wurmbrand 2001, 2004, 2007); por esta razón, y de acuerdo con lo expuesto en los apartados anteriores, la estructura es transparente para el ascenso de clíticos, constituye un único dominio para la asignación de caso, cuenta con una sola posición — sobre hacer - para los modales epistémicos, y con un único ST, lo cual impide que el infinitivo pueda pasivizar y que su AE reciba caso nominativo:

$$
\begin{aligned}
& {\left[_ { \mathrm { ST } } \mathrm { SD } _ { \mathrm { i } } [ \mathrm { T } ] \left[_ { \mathrm { SM } \text { -epistémico } } [ \mathrm { M } ] \left[_ { \mathrm { SAsp } } [ \mathrm { Asp } ] \left[_ { \mathrm { SVoz } } h _ { \mathrm { i } } [ \mathrm { Voz } ] \left[_ { \mathrm { SM } \text { -deontico } } [ \mathrm { M } ] \left[_{\mathrm{Sv}}[\mathrm{v} \text { hacer }]\right.\right.\right.\right.\right.\right.}
\end{aligned}
$$

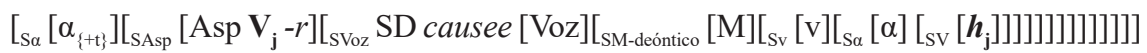

Las diferencias entre los distintos tipos de PPCC en las lenguas radican, entre otros factores, en el tamaño de la estructura inferior o subordinada. En el caso concreto de las CCAA del español, se ha defendido en estas páginas que el infinitivo no se encuentra en un SV escueto ( $c f r$. López 2001), sino que proyecta cierta estructura funcional, incluyendo SVoz, SM-deóntico y SAsp.

El infinitivo proyecta su estructura argumental completa, incluyendo el AE. En (77) vemos este argumento situado en $\mathrm{SVoz}^{40}$, de acuerdo con lo expuesto en el apartado 2.2. De este modo, las CCAA tienen dos posiciones para los adverbios orientados al agente (78a) y pueden ser recursivas (74b). Concretamente, la recursividad se produce gracias a que hacer es capaz de subordinar una estructura idéntica a la suya propia, es decir, si Yuridia se encuentra en Voz como AE del verbo flexionado,

$40 \quad$ Wurmbrand (2001) considera que las CCAA no son construcciones de reestructuración precisamente porque tienen Voz, es decir, porque el infinitivo no está en un SV escueto. No obstante, la misma autora defiende en su trabajo de 2015(b) que ciertos verbos de reestructuración pueden incluir esta proyección. En la misma línea, el análisis aquí presentado asume que puede haber reestructuración siempre y cuando no haya ST en el infinitivo. 
Macarena, Luis, Javi y Alex deben encontrarse en sus respectivas proyecciones Voz como AAEE de los infinitivos para que la recursividad sea posible:

(78) a. Víctor ${ }_{\mathrm{i}}$ hizo trabajar a Sara duramente $_{\mathrm{ij}}$ en el ensayo.

b. $\left[_{\mathrm{SVoz}}\right.$ Yuridia $\left[_{\mathrm{SV}}\right.$ hizo $\left[_{\mathrm{SVoz}}\right.$ a Macarena $\left[_{\mathrm{SV}}\right.$ hacer $\left[_{\mathrm{SVoz}}\right.$ a Luis $\left[_{\mathrm{SV}}\right.$ hacer $\left[_{\mathrm{SVoz}}\right.$ a Javi $\left[_{\mathrm{SV}}\right.$ hacer [svoz a Alex [sv traer vodka]]]]]]]]

En cuanto al orden de palabras, encontramos en la bibliografía tres posibles explicaciones. La primera es que el SV asciende por encima de su sujeto bien a una proyección funcional, bien al SV de hacer, de acuerdo con las versiones estándar del predicate raising (Kayne 1975; Burzio 1986; Baker 1988) y con las versiones actualizadas (Torrego 2010; Campanini \& Pitteroff 2013; Ordóñez \& Saab 2017). La segunda postula que el especificador donde se proyecta el sujeto del infinitivo se encuentra a la derecha (Guasti 1996; Folli \& Harley 2007). Por último, la tercera propone que el causee se encuentra en una aplicativa (Ippolito 2000; Torrego 2010; Campanini \& Pitteroff 2013).

Tras rechazar las dos últimas hipótesis en el apartado 2, el análisis de ascenso del infinitivo es el único disponible ${ }^{41}$. De acuerdo con la estructura de (77), el infinitivo no llega a introducirse en la proyección de hacer, sino que se desplaza, para incorporarse a la desinencia $-r$, a su propio SAsp, una proyección funcional intermedia que no constituye una barrera oracional ${ }^{42}$. Hacer y el infinitivo no se encuentran bajo un mismo núcleo $\mathrm{V}$ —no hay incorporación— ${ }^{43}$, ni dentro de un mismo SV — no hay predicate raising en sentido estricto-, lo cual explica de manera natural todas las propiedades descritas en los apartados anteriores.

Nótese que, si se prescindiera de estructura funcional en el infinitivo, resultaría necesario explicar que hacer seleccionara SVoz en unas ocasiones (79a) y Sv en otras (79b). En cambio, de acuerdo con (77), hacer selecciona siempre el mismo tipo de complemento, SAsp.

(79) a. Andrea [hizo [SVoz Diana [Sv [v reír]]]]

b. Nerea [hizo [Sv [v desaparecer [SV [V] [SD las pruebas]]]]]

${ }_{41} \quad$ Nótese que la postulación original del predicate raising asume que las CCAA han de ser construcciones derivadas, mientras que en nuestra propuesta las CCAA son monoclausales a lo largo de la derivación.

42 Puesto que SAsp es la posición funcional más alta de la estructura subordinada, no hay ninguna otra que pueda intervenir entre hacer y el infinitivo.

43 Véase Kayne (1975), Baker (1988) e Ippolito (2000) para una discusión más detallada en contra de los análisis de incorporación. El argumento más evidente, además de los aquí revisados, es que hacer y el infinitivo son unidades léxicas distintas, es decir, no hay incorporación morfofonética. 
La presencia de SAsp tiene ciertas consecuencias para el estatus de hacer. Recordemos que el elemento jerárquicamente más alto en un PC puede ser de naturaleza léxica, funcional o cuasifuncional (Cardinaletti 2004). Algunos autores defienden que los verbos de reestructuración son elementos funcionales (c $f r$. Cinque 2001), mientras otros defienden que pueden ser tanto funcionales como léxicos ( $c f r$. Wurmbrand 2004). Hacer, concretamente, ha de ser un elemento más léxico que funcional, pues de lo contrario se esperaría que fuera capaz de combinarse directamente con una estructura menor que SAsp, o incluso con un SV escueto.

A lo largo de este trabajo se han observado varias propiedades de las CCAA que apuntan a un estatus léxico —o, al menos, cuasiléxico ${ }^{44}$ - de hacer. En primer lugar, a diferencia de faire y fare, hacer es capaz de subordinar un SC:

(80) Andrea hizo que Diana llorara de risa.

Una posible explicación para (80) es que hacer, como verbo causativo, es unas veces un elemento funcional - cuando selecciona un infinitivo- y otras un elemento léxico pleno, capaz de subordinar un SC. Sin embargo, un análisis uniforme de hacer resulta más elegante, pues el verbo mantiene su estatus al seleccionar complementos que, a pesar de tener distinto tamaño, cuentan siempre con cierta estructura funcional -no son SSVV escuetos-.

En segundo lugar, la recursividad de las CCAA (78b) demuestra que el propio hacer puede funcionar como el verbo subordinado semánticamente pleno. De hecho, hacer establece relaciones temáticas con su AE y aporta un significado semántico pleno, propiedades que Cardinaletti (2004) y Wurmbrand (2004) señalan como características de los verbos léxicos de reestructuración. Además, hacer, a diferencia de faire, puede pasivizar, lo cual no es esperable si se trata de un elemento funcional (Wurmbrand 2004; Campanini \& Pitteroff 2013).

La posibilidad de interpolar distintos elementos entre el verbo flexionado y el infinitivo es otro argumento a favor del estatus léxico de hacer, pues, si se tratara de un elemento funcional, sería esperable que presentara mayor grado de fusión con el infinitivo.

Volviendo al orden de palabras, queda por resolver el problema de la anteposición del causee. Como quedó explicado en el apartado 2, formular una hipótesis sobre la posición a la que se desplaza el causee supera los propósitos de este trabajo;

44 De acuerdo con un revisor anónimo, uno de los factores que otorgan a hacer un estatus cuasi-léxico es el hecho de que no impone a sus complementos las severas restricciones léxicas que imponen otros verbos ligeros. 
sin embargo, para cerrar este apartado cabe realizar una breve reflexión al respecto. Los datos que han ido surgiendo a lo largo de estas páginas permiten aislar las siguientes características de los causees antepuestos: en primer lugar, los causees inanimados resisten la anteposición - más concretamente, de acuerdo con Ordóñez y Saab (2017), esta está ligada al MDO- En segundo lugar, la anteposición se ve favorecida por factores relacionados con el procesamiento, volviéndose casi obligatoria en contextos en los que el peso sintáctico del SV, el encadenamiento de varios infinitivos o el encadenamiento de varios SSDD hacen necesario reordenar las palabras para facilitar la comprensión. En tercer lugar, la anteposición puede estar relacionada con la estructura informativa. De acuerdo con (74) la posición de aterrizaje más plausible para el causee antepuesto es el especificador del $\mathrm{S} \alpha$ de hacer, que le asigna caso. Puesto que el movimiento es opcional - el SD recibe caso a distancia-, da lugar a una interpretación informativamente marcada. La postulación de la proyección Sa viene inspirada por López (2012), quien la emplea para explicar el MDO, y se fundamenta en la necesidad de separar la información relativa a la presencia / ausencia de $\mathrm{AE}$ del núcleo v, cuya función es únicamente verbalizadora (Vivanco 2016). Por lo tanto, un análisis en esta línea sería compatible con la propuesta de anteposición mediante revoltijo (scrambling) vinculada al MDO que defienden Ordóñez y Saab (2017).

\section{CONCLUSIONES}

Los PPCC conforman una clase heterogénea que abarca prácticamente cualquier oración simple en la que se integren dos núcleos semánticos. Los verbos de reestructuración, a su vez, conforman también una clase heterogénea tanto dentro de una lengua como a nivel tipológico. Así, hacer no comparte las propiedades de otros verbos de reestructuración (intentar, ver, odiar, etc.) y no funciona exactamente igual que sus homólogos en otras lenguas (fare, faire, fazer, etc.). Por ello este trabajo se ha centrado exclusivamente en las CCAA del español, ofreciendo un detallado panorama general de sus propiedades a fin de esclarecer algunos de los puntos más controvertidos para su análisis.

Las CCAA son estructuras monoclausales desde el comienzo de la derivación, por lo que cualquier operación de reducción resulta innecesaria. Esto no implica, sin embargo, que el infinitivo haya de encontrarse en un SV escueto, pues puede proyectar SVoz, SM-deóntico y SAsp, como ha quedado demostrado empíricamente en los apartados 2.2., 3.4. y 3.5. Además de contribuir a resolver el problema del tamaño del infinitivo, la presencia de SVoz responde al debate teórico sobre la posición de base del causee, y la presencia de SAsp, a su vez, permite un análisis de ascenso que explica el orden no marcado de palabras en el que el causee sigue al SV. 
En cuanto al estatus de hacer, los datos aquí estudiados apuntan a que se trata de un elemento cuasifuncional, pues preserva varias características propias de los verbos léxicos plenos. Esta parece ser una de las claves para establecer las diferencias entre hacer y sus contrapartidas en otras lenguas.

Este trabajo ha abordado así mismo otras cuestiones, como la interpretación semántica de las CCAA y la asignación de caso. Así pues, se ha argumentado que las dos posibles lecturas de estas construcciones no son el producto de dos estructuras sintácticas distintas y no se ven afectadas tampoco por el orden de palabras, sino que son meramente el resultado de la interpretación composicional de los argumentos. En cuanto al caso, se ha planteado un análisis de asignación por defecto en la línea de Marantz (1991), haciendo uso de la proyección funcional $\mathrm{S} \alpha$.

Para terminar, han quedado aquí planteados para una futura investigación dos problemas relacionados con el tipo de estructuras que puede subordinar hacer: los verbos psicológicos con experimentante dativo y las construcciones anticausativas. Un estudio más detallado sobre estas cuestiones contribuiría a lograr un conocimiento más profundo sobre la sintaxis de las CCAA y las razones que subyacen a los contrastes presentados.

\section{BIBLIOGRAFÍA}

Aissen, J. \& D. Perlmutter, D. (1983): «Clause reduction in Spanish», en D. Perlmutter (ed.): Studies in Relational Grammar 1. Chicago: The University of Chicago Press, pp. 360-403.

Alarcos, E. (1970): Estudios de gramática funcional del Español. Madrid: Gredos. Alexiadou, A. \& E. Anagnostopoulou \& F. Schäfer (2006): «The properties of anticausatives crosslinguistically», en M. Frascarelli (ed.): Phases of Interpretation. Berlin: Mouton, pp. 187-211. https://doi.org/10.1515/9783110197723.4.187

Alexiadou, A. \& E. Anagnostopoulou \& F. SchäFer (2015): External Arguments in Transitivity Alternations. A Layering Approach. Oxford: Oxford University Press. https://doi.org/10.1093/acprof:oso/9780199571949.001.0001

Alsina, A. (1992): «On the argument structure of causatives», Linguistic Inquiry 23, pp. 517-555.

BAKER, M. (1988): Incorporation: A Theory of Grammatical Function Changing. Chicago: University of Chicago Press.

Bello, A. (1984 [1847]): Gramática de la lengua castellana destinada a los americanos. Madrid: EDAF.

Bordelois, I. (1988): «Causatives: From lexicon to syntax», Natural Language and Linguistic Theory 6, pp. 57-93. https://doi.org/10.1007/bf01791592 
Brennan, V. M. (1993): Root and Epistemic Modal Auxiliary Verbs. Boston: University of Massachussets. Doctoral Dissertations available from Proquest. Paper AAI9316625. http://scholarworks.umass.edu/dissertations/AAI9316625 [Consulta: 03/02/2015].

Burzio, L. (1986): Italian Syntax: A Government and Binding Approach. Dordrecht: Reidel. https://doi.org/10.1007/978-94-009-4522-7

ButT, M. (1995): The structure of Complex Predicates in Urdu. Standford: CSLI Publications.

Campanini, C. \& M. Pitteroff (2013): «Analytic causatives: A German-Italian comparative approach», en E. Boone \& M. Kohlberger \& M. Schulpen (eds.): Proceedings of ConSOLE XX. Leiden: Leiden University, pp. 45-70.

Cano Aguilar (1977): «Las construcciones causativas en español», BRAE 57, pp. 231-351.

CARdinaletti, A. (2004): «Clitic positions and restructuring in Italian», Linguistic Inquiry 35(4), pp. 519-557. https://doi.org/10.1162/0024389042350523

CARrasco GutiÉrRez, A. (2017): «Sobre la sintaxis de la percepción no epistémica», en S. Gumiel \& M. Leonetti \& I. Pérez Jiménez (eds.): Investigaciones actuales en lingüistica. Volumen 3: sintaxis. Madrid: Universidad de Alcalá, pp. 31-46.

Cinque, G. (1999): Adverbs and functional heads: A cross-linguistics perspective. Oxford: Oxford University Press.

CINQUe, G. (2001): «Restructuring and the order of aspectual and root modals heads», en G. Cinque \& G. Salvi (eds.): Current Studies in Italian Syntax. Amsterdam: Elsevier, pp. 137-155.

CInQue, G. (2006): Restructuring and functional heads. The Cartography of Syntactic Structures. Vol. 4. Oxford: Oxford University Press.

Ciutescu, E. (2013): «Micro-parametric variation in Romance causative constructions», en A. Cornilescu (ed.): Bucarest Working Papers in Linguistics XV (2). Bucarest: Universitatea din Bucareşti, pp. 46-60.

CiUtesCu, E. (2015): «Romance causatives and object shift», en E. Aboh \& J. Schaeffer \& P. Sleeman (eds.): Romance Languages and Linguistic Theory 2013. Amsterdam: John Benjamins, pp. 21-38. https://doi.org/10.1075/rllt.8.02ciu

Comrie, B. (1976): «The syntax of causative constructions: cross-language similarities and divergences», en M. Shibatani (ed.): Syntax and semantics 6: The grammar of Causative Constructions. New York: Academic Press, pp. 261-312.

Cuervo, M. C. (2003): Datives at Large. Boston: Massachusetts Institute of Technology [tesis doctoral]. 
Cuervo, M. C. (2016): «Disappearing se in predicates embedded under causative light verbs». Presentación en el Workshop on Romance se / si. University of Wisconsin-Madison, April 21 $1^{\text {st }}-22^{\text {nd }}, 2016$.

FARMER, A. K. (1980): On the Interaction of Morphology and Syntax. Boston: Massachusetts Institute of Technology [tesis doctoral].

Felser, C. (1999): Verbal Complement Clauses. A Minimalist Study of Direct Perception Construction. Amsterdam: John Benjamins. https://doi.org/10.1075/la.25

Fernández Lagunilla, M. \& F. Dios López (1991): «Dos análisis gramaticales de ciertas construcciones completivas de infinitivo en español: a propósito de los verbos causativos y de percepción», Revista de la Sociedad Española de Lingüistica 21(2), pp. 217-232.

FoDOR, J. (1970): «Three reasons for not deriving 'kill' from 'cause to die'», Linguistic Inquiry 1, pp. 29-38.

Folli, R. \& H. HaRley (2005): «Flavors of $v$ : Consuming results in Italian and English», en R. Slabakova \& P. Kempchinsky (eds.): Aspectual Inquiries. Dordrecht: Kluwer, pp. 95-120. https://doi.org/10.1007/1-4020-3033-9_5

Folli, R. \& H. HARLey (2007): «Causation, obligation, and argument structure: on the nature of little v», Linguistic Inquiry 38, pp. 197-238. https://doi.org/10.1162/ ling.2007.38.2.197

Gallego, A. J. (2013): «Object shift in Romance», Natural Language and Linguistic Theory 31, pp. 409-451. https://doi.org/10.1007/s11049-013-9188-6

Geach, P. T. (1970): «A program for syntax», Synthese 22, pp. 3-17.

Grimshaw, J. \& R. A. Mester (1985): «Complex Verb Formation in Eskimo», Natural Language and Linguistic Theory 3, pp. 1-19. https://doi.org/10.1007/bf00205412 Guasti, M. T. (1993): Causative and Perception Verbs. Torino: Rosenberg and Sellier.

GuASTI, M. T. (1996): «Semantic restrictions in Romance causatives and the incorporation approach», Linguistic Inquiry 27, pp. 294-313.

HaCQuard, V. (2006): Aspects of Modality. Boston: Massachusetts Institute of Technology [tesis doctoral].

HACQUARD, V. (2009): «On the interaction of aspect and modal auxiliaries», Linguistics and Philosophy 32, pp. 279-312. https://doi.org/10.1007/s10988-009-9061-6

Haegeman, L. \& H. van RiemsdiJK (1986): «Verb Projection Raising, Scope, and the Typology of Rules Affecting Verbs», Linguistic Inquiry 17, pp. 417-466.

HALE, K. \& S. J. Keyser (1993): «On argument structure and the lexical expression of syntactic relations», en K. Hale \& S. J. Keyser (eds.): The View from Building 20. Cambridge, Mass.: MIT Press, pp. 53-109. 
Hale, K. \& S. J. Keyser (2002): Prolegomenon to a Theory of Argument Structure. Cambridge, Mass.: MIT Press. https://doi.org/10.7551/mitpress/5634.001.0001

Harley, H. (1995): Subjects, Events and Licensing. Boston: Massachusetts Institute of Technology [tesis doctoral].

Hernanz, M. L. (1982): El infinitivo en español. Barcelona: Universidad Autónoma de Barcelona.

Hernanz, M. L. (1999): «El infinitivo», en I. Bosque \& V. Demonte (eds.): Gramática descriptiva de la lengua española. Madrid: Espasa Calpe, pp. 2196-2356.

Higginbotham, J. (2000): «On events in linguistic semantics», en J. Hgginbotham \& F. Pianersi \& A. Varzi (eds.): Speaking of Events. Oxford: Oxford University Press, pp. 53-83.

IgLesIAS BANGO, M. (1992): «Acerca del supuesto estatuto perifrástico de la construcción causativa hacer + infinitivo y otras cuestiones conexas (I y II)», Contextos X, 19/20, pp. 87-148.

InOve, K. (1969): A study of Japanese Syntax. The Hague: Mouton.

IpPolito, M. (2000): Remarks on the Argument Structure of Romance Causatives. Ms. Boston: Massachusetts Institute of Technology.

JACKENDOFF, R. (1972): Semantic Interpretation in Generative Grammar. Cambridge, Mass.: MIT Press.

KaYne, R. (1975): French Syntax. Cambridge, Mass.: MIT Press.

KaYne, R. (2005): Movement and Silence. Oxford: Oxford University Press. https:// doi.org/10.1093/acprof:oso/9780195179163.001.0001

KeY, G. (2013): The Morphosyntax of the Turkish Causative Construction. Tucson: University of Arizona [tesis doctoral].

KIM, K. (2012): «Argument structure licensing and English have"», Journal of Linguistics 48(1), pp. 71-105. https://doi.org/10.1017/S0022226711000168

Kuno, S. (1973): The structure of the Japanese language. Cambridge, Mass.: MIT Press.

KuRodA, S. Y. (1965): «Causative forms in Japanese», Foundations of Language 1, pp. $30-50$.

LABelLe, M. (1992): «Change of state and valency», Journal of Linguistics 28, pp. 375-414. https://doi.org/10.1017/S0022226700015267

Levin, B. \& M. RAPPAPORT Hovav (1999): «Two structures for compositionally derived events». Proceedings of SALT 9. Ithaca, New York: Cornell Linguistics Circle Publications, Cornell University, pp. 199-223. https://doi.org/10.3765/salt. v9i0.2836 
LóPEz, L. (2001): «The cause and the theory of bare phrase structure», en J. Gutiérrez Rexach \& L. Silva Villar (eds.): Current issues in Spanish syntax and semantics. Berlin: Mouton de Gruyter, pp. 221-241.

LóPEz, L. (2012): Indefinite Objects. Scrambling, Choice Functions and Differential Marking. Linguistic Inquiry Monograph 63. Cambridge, Mass.: MIT Press. https:// doi.org/10.7551/mitpress/9165.001.0001

Manning, D. C. \& I. A. SAG \& M. IIDA (1999): «The lexical integrity of Japanese causatives», en R. D. Levine \& G. M. Green (eds.): Studies in Contemporary Phrase Structure Grammar. Cambridge: Cambridge University Press, pp. 39-79. https://doi.org/10.1017/CBO9780511554421.002

Marantz, A. (1991): «Case and Licensing», en G. F. Westphal \& B. Ao \& H-R. Chae (eds.): ESCOL'91 Proceedings of the English Eastern States Conference on Linguistics. Columbus: Ohio State University, Department of Linguistics, pp. 234-253.

Marín, R. (2011): «Casi todos los predicados psicológicos son estativos», en A. Carrasco (ed.): Sobre estados y estatividad (Lincom Studies in Theoretical Linguistics, vol. 46). München: Lincom, pp. 26-44.

Meurers, W. D. (2000): Lexical Generalizations in the Syntax of German Non-finite Constructions. Tübingen: Universität Tübingen [tesis doctoral].

Miyagawa, S. (1980): Complex Verbs and the Lexicon. Tucson: University of Arizona [tesis doctoral].

Moreno Cabrera, J. C. (1987): Fundamentos de sintaxis general. Madrid: Síntesis.

Moreno Cabrera, J. C. (2003): Semántica y gramática. Sucesos, papeles temáticos y relaciones sintácticas. Madrid: Antonio Machado.

Morimoto, Y. (2011): «El control en los predicados estativos», A. Carrasco Gutiérrez (ed.): Sobre estados y estatividad. München: Lincom Europa, pp. 122-141.

NASH, L. (2006): «Structuring VP: goals». EALING Lectures, Septiembre 2006, École Normale Superieur.

Nedjalkov, V. P. \& G. G. SiLnitsky (1973): «The typology of morphological and lexical causatives», en F. Kiefer (ed.): Trends in soviet Theoretical Linguistics. Dordrecht: Reidel, pp. 1-32. https://doi.org/10.1007/978-94-010-2536-2_1

Neeleman, A. \& H. van De Koot (2012): «The linguistic expression of causation», en M. Everaert \& T. Siloni \& M. Marelj (eds.): The Theta System: Argument Structure at the Interface. Oxford: Oxford University Press, pp. 20-51. https:// doi.org/10.1093/acprof:oso/9780199602513.003.0002

Noyer, R. (1997): Features, Positions and Affixes in Autonomous Morphological Structure. New York: Garland. 
OrdóÑez, F. (1998): «Postverbal asymetries in Spanish», Natural Language and Linguistic Theory 16, pp. 313-346. https://doi.org/10.1023/A:1006051703562

ORDóÑEZ, F. \& A. SAAB (2017): «Sobre la distribución de los sujetos causados en los dialectos del español», Estudos linguísticos e literários 58, pp. 186-209. https:// doi.org/10.9771/ell.v0i58.26811

PiCAllo, M. C. (1990): «Modal verbs in Catalan», Natural Language and Linguistic Theory 8, pp. 285-312. https://doi.org/10.1007/bf00208525

PYLKKÄNEN, L. (2008): Introducing Arguments. Cambridge, Mass.: MIT Press. https:// doi.org/10.7551/mitpress/9780262162548.001.0001

Ramchand, G. (2008): Verb Meaning and the Lexicon: A First Phase Syntax. Cambridge, Mass.: Cambridge University Press. https://doi.org/10.1017/cbo9780511486319

Real Academia Española (1973): Esbozo de una nueva gramática de la lengua española. Madrid: Espasa Calpe.

Real ACADEmia EsPañola: Corpus de referencia del español actual (CREA). http:// corpus.rae.es/creanet.html [última consulta: mayo 2016].

Real Academia de la Lengua Española (2009): Nueva Gramática de la Lengua Española. Madrid: Espasa Libros.

Richards, N. (2010): Uttering Trees. Cambridge, Mass.: MIT Press. https://doi. org/10.7551/mitpress/9780262013765.001.0001

RIzzI, L. (1978): «A restructuring rule in Italian syntax», en S. J. Keyser (ed.): Recent Transformational Studies in European Languages. Cambridge, Mass.: MIT Press, pp. 113-158.

RizzI, L. (1982): Issues in Italian Syntax. Dordrecht: Foris. https://doi. org/10.1515/9783110883718

RoBERTS, I. (1997): «Restructuring, head movement and locality», Linguistic Inquiry 28, pp. 423-460.

SAito, M. \& H. Hoshi (2000): «The Japanese light verb construction and the Minimalist Program», en R. Martin \& D. Michaels \& J. Uriaguereka (eds.): Step by Step: Essays on Minimalis Syntax in Honor of Howard Lasnik. Cambridge Massachusetts: MIT Press, pp. 261-295.

SchäFER, F. (2008): The syntax of (anti-)causatives. External Arguments in Change of State Contexts. Amsterdam: John Benjamins. https://doi.org/10.1075/la.126

Shibatani, M. (1973): A Linguistics Study of Causative Constructions. Berkeley: University of California [tesis doctoral].

SoARES DA SilvA, A. (2012): «Stages of grammaticalization of causative verbs and constructions in Portuguese, Spanish, French and Italian», Folia Linguistica 46(2), pp. 1614-7608. https://doi.org/10.1515/flin.2012.018 
Sportiche, D. (1988): «A theory of floating quantifiers and its corollaries for constituent structure», Linguistic Inquiry 19, pp. 33-60.

Stechow, A. vON (1990): «Focusing and backgrounding operators», en W. Abraham (ed.): Discourse Particles. Amsterdam: John Benjamins, pp. 37-84. https://doi. org/10.1075/pbns.12.04ste

SvenONIUS, P. (2008): «Complex predicates and the functional sequence», Nordlyd (Tromsø working papers in linguistics) 35(1), pp. 49-88. https://doi.org/10.7557/12.137 Torrego, E. (2010): «Variability in the case patterns of causative formation in Romance and its implications», Linguistic Inquiry 41(3), pp. 445-470. https://doi. org/10.1162/ling_a_00004

Treviño, E. (1992): «Subjects in Spanish causative constructions», en P. Hirschbüler \& K. Koerner (eds.): Romance Languages and Mordern Linguistic Theory. Amsterdam: John Benjamins, pp. 309-324. https://doi.org/10.1075/cilt.91.19tre

Treviño, E. (1994): Las causativas del español con complemento de infinitivo. México: El Colegio de México.

Tubino Blanco, M. \& H. Harley \& J. Haugen (2014): «Affixal light verbs and complex predicated in Hiaki», en H. Kishimoto \& Y. Yumoto (eds.): Current Issues in Complex Predicate Research. Tokyo: Hituzi Syobo, pp. 257-290.

Vivanco, M. (2016): Causatividad y cambio de estado en español. La alternancia causativo-inacusativa. Madrid: Universidad Complutense de Madrid [tesis doctoral].

Williams, E. (2003): Representation Theory. Cambridge, M.A.: MIT Press.

Wurmbrand, S. (2001): Infinitives. Restructuring and Clause Structure. Berlin: Mouton de Gruyter.

WuRMBrAND, S. (2004): «Two types of restructuring: Lexical vs. functional», Lingua 114(8), pp. 991-10014. https://doi.org/10.1016/s0024-3841(03)00102-5

Wurmbrand, S. (2007): «How complex are complex predicates», Syntax 10, pp. 243-248. https://doi.org/10.1111/j.1467-9612.2007.00110.x

Wurmbrand, S. (2015a): «Restructuring cross-linguistically», en T. Bui \& D. Özyildiz (eds.): Proceedings of the North Eastern Linguistics Society Annual Meeting 45. Amherst: University of Massachussets, GLSA, pp. 227-240.

Wurmbrand, S. (2015b): «Complex predicate formation via Voice incorporation», en L. Nash \& P. Samvelian (eds.): Approaches to Complex Predicates. Leiden: Brill, pp. 248-290. https://doi.org/10.1163/9789004307094_010

ZubiZARRETA, M. L. (1985): «The relation between morphophonology and morphosyntax: the case of Romance causatives», Linguistic Inquiry 16(2), pp. 247-288. 